\title{
The Cherenkov Telescope Array potential for the study of young supernova remnants
}
B. S. Acharya ${ }^{\text {bk }}$, C. Aramo ${ }^{\text {bu }}$, A. Babic ${ }^{\text {aa }}$, J. A. Barrio ${ }^{\text {eq }}$, A. Baushev ${ }^{\text {bd }}$, J. Becker Tjus ${ }^{\text {ba }}$, D. Berge ${ }^{\text {dt }}$, M. Bohacova ${ }^{\text {ac }}$, A. Bonardi ${ }^{\text {au }}$, A. Brown ${ }^{\text {fe }}$, V. Bugaev ${ }^{\text {gi }}$, T. Bulik ${ }^{\text {ed }}$, M. Burton ${ }^{1}$, G. Busetto ${ }^{\text {cl }}$, P. Caraveo ${ }^{\text {bq }}$, R. Carosi ${ }^{\text {bw }}$, J. Carr ${ }^{\text {al }}$, P. Chadwick ${ }^{\text {fe }}$, J. Chudoba ${ }^{\text {ac }}$, V. Conforti ${ }^{\text {bp }}$, V. Connaughton ${ }^{\text {fx }}$, J. L. Contreras ${ }^{\mathrm{eq}}$, G. Cotter ${ }^{\mathrm{fk}}$, F. Dazzi ${ }^{\mathrm{cl}}$, A. De Franco ${ }^{\mathrm{fk}}$, I. de la Calle ${ }^{\mathrm{eq}}$, R. de los Reyes Lopez ${ }^{\text {aw }}$, B. De Lotto ${ }^{\mathrm{cm}}$, F. De Palma ${ }^{\text {bs }}$, T. Di Girolamo ${ }^{\text {bu }}$, C. Di Giulio ${ }^{\text {by }}$, F. Di Pierro ${ }^{\text {ci }}$, J.-L. Dournaux ${ }^{\text {as }}$, V. Dwarkadas ${ }^{\mathrm{fz}}$, J. Ebr ${ }^{\text {ac }}$, K. Egberts ${ }^{\mathrm{o}}$, M. Fesquet ${ }^{\mathrm{aj}}$, H. Fleischhack ${ }^{\mathrm{at}}$, L. Font ${ }^{\mathrm{ep}}$, G. Fontaine ${ }^{\mathrm{ap}}$, A. Förster ${ }^{\text {aw }}$, M. Fuessling ${ }^{\text {bd }}$, B. Garcia ${ }^{\text {b }}$, R. Garcia López ${ }^{\text {ek }}$, M. Garczarczyk ${ }^{\text {at }}$, F. Gargano ${ }^{\text {bs }}$, D. Garrido ${ }^{\text {ep }}$, M. Gaug ${ }^{\text {ep }}$, N. Giglietto ${ }^{\text {bs }}$, F. Giordano ${ }^{\text {bs }}$,

A. Giuliani ${ }^{\mathrm{bq}}$, N. Godinovic ${ }^{\mathrm{z}}$, M. M. Gonzalez ${ }^{\mathrm{dq}}$, T. Grabarczyk ${ }^{\mathrm{dw}}$, T. Hassan ${ }^{\mathrm{eq}}$, J. Hörandel ${ }^{\mathrm{ds}}$, M. Hrabovsky ${ }^{\text {ac }}$, D. Hrupec ${ }^{\text {aa }}$, T. B. Humensky ${ }^{\text {fr }}$, J. Huovelin ${ }^{\text {af }}$, M. Jamrozy ${ }^{\mathrm{eb}}$, P. Janecek ${ }^{\mathrm{ac}}$, P. E. Kaaret ${ }^{\mathrm{gb}}$, U. Katz ${ }^{\mathrm{bb}}$, S. Kaufmann ${ }^{\mathrm{av}}$, B. Khélifi ap,ai , W. Kluźniak ${ }^{\text {dy }}$, J. Kocot ${ }^{\text {dw }}$, N. Komin ${ }^{\text {ao }}$, H. Kubo ${ }^{\text {cq }}$, J. Kushida ${ }^{\text {dh }}$, G. Lamanna ${ }^{\mathrm{ao}}$, W. H. Lee ${ }^{\mathrm{dq}}$, J.-P. Lenain ${ }^{\mathrm{aq}}$, T. Lohse ${ }^{\mathrm{az}}$, S. Lombardi ${ }^{\mathrm{cg}}$, R. López-Coto ${ }^{\text {en }}$, A. López-Oramas ${ }^{\text {en }}$, F. Lucarelli ${ }^{\mathrm{cg}}$, M. C. Maccarone ${ }^{\mathrm{br}}$, G. Maier $^{\text {at }}$, P. Majumdar ${ }^{\text {bj }}$, G. Malagutib ${ }^{\text {bp }}$, D. Mandat ${ }^{\text {ac }}$, M. N. Mazziotta ${ }^{\text {bs }}$, K. Meagher ${ }^{\mathrm{fs}}$, N. Mirabal ${ }^{\mathrm{eq}}$, A. Morselliby ${ }^{\text {by }}$ E. Moulin ${ }^{\mathrm{aj}}$, J. Niemiec ${ }^{\mathrm{dx}}$,

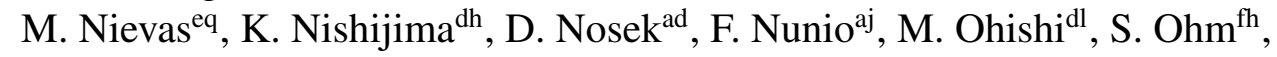
R. A. Ong ${ }^{\mathrm{gl}}$, R. Orito ${ }^{\mathrm{dj}}$, N. Otte ${ }^{\mathrm{fs}}$, M. Palatka ${ }^{\mathrm{ac}}$, G. Pareschi ${ }^{\mathrm{cd}}$, M. Pech ${ }^{\mathrm{ac}}$, M. Persic ${ }^{\text {ch,cm }}$, M. Pohl ${ }^{\text {bd }}$, M. Prouza ${ }^{\text {ac }}$, A. Quirrenbach ${ }^{\text {av }}$, S. Rainò ${ }^{\text {bs }}$, G. Rodriguez Fernandez ${ }^{\text {by }}$, P. Romano ${ }^{\text {br }}$, A. C. Rovero ${ }^{\text {d }}$, B. Rudak ${ }^{\text {dy }}$, P. Schovanek ${ }^{\text {ac }}$, M. Shayduk ${ }^{\text {at,* }}$, H. Siejkowskid ${ }^{\text {dw }}$, A. Sillanpää ${ }^{\text {ah }}$, S. Stefanik ${ }^{\text {ad }}$, T. Stolarczyk ${ }^{\mathrm{aj}}$, M. Szanecki ${ }^{\mathrm{ec}}$, T. Szepieniec ${ }^{\mathrm{dw}}$, L. A. Tejedor ${ }^{\mathrm{eq}}$, I. Telezhinsky ${ }^{\text {bd }, * *}$, M. Teshima ${ }^{\text {ax,dl }}$, L. Tibaldo ${ }^{\text {fw }}$, O. Tibolla ${ }^{\text {be }}$, G. Tovmassian ${ }^{\mathrm{dq}}$, P. Travnicek ${ }^{\mathrm{ac}}$, M. Trzeciak ${ }^{\mathrm{dw}}$, P. Vallania ${ }^{\mathrm{ci}}$, C. van Eldik ${ }^{\mathrm{bb}}, \mathrm{S}$. Vercellone ${ }^{\mathrm{br}}$, C. Vigorito ${ }^{\text {bz }}$, S. J. Wagner ${ }^{\text {av }}$, S. P. Wakely ${ }^{\text {fz }}$, A. Weinstein ${ }^{\text {gc }}$, A. Wierzcholska ${ }^{\text {eb }}$, A. Wilhelm ${ }^{\text {bd }}$, P. Wojcik ${ }^{\mathrm{dw}}$, T. Yoshikoshi ${ }^{\mathrm{dl}}$

${ }^{a}$ Centro de Investigaciones en Láseres y Aplicaciones (CEILAP - CITEFA / CONICET), Argentina

${ }^{b}$ Instituto de Tecnologias en Deteccion y Astroparticulas (CNEA / CONICET / UNSAM), Argentina

${ }^{c}$ Centro Atómico Bariloche (CNEA-CONICET-IB/UNCUYO), Argentina

${ }^{d}$ Instituto de Astronomía y Física del Espacio (IAFE CONICET-UBA), Argentina

${ }^{e}$ Instituto Argentino de Radioastronomía (CCT La Plata - CONICET), Argentina 


\author{
${ }^{f}$ Instituto de Física Rosario, Argentina \\ ${ }^{g}$ UID GEMA - Departamento de Aeronáutica (Facultad de Ingeniería, UNLP), Argentina \\ ${ }^{h}$ Alikhanyan National Science Laboratory, Yerevan Physics Institute, Armenia \\ ${ }^{i}$ Australian National University, Australia \\ ${ }^{j}$ University of Adelaide, Australia \\ ${ }^{k}$ Monash University, Australia \\ ${ }^{l}$ University of New South Wales, Australia \\ ${ }^{m}$ University of Sydney, Australia \\ ${ }^{n}$ University of Western Sydney, Australia \\ ${ }^{o}$ Institut für Astro- und Teilchenphysik, Leopold-Franzens-Universität, Austria \\ ${ }^{p}$ Centro Brasileiro de Pesquisas Físicas, Brazil \\ ${ }^{q}$ Centro de Ciências Naturais e Humanas - Universidade Federal do ABC, Brazil \\ ${ }^{r}$ Instituto de Astronomia, Geofísico, e Ciências Atmosféricas, Brazil \\ ${ }^{s}$ Instituto de Física - Universidade de São Paulo, Brazil \\ ${ }^{t}$ Instituto de Física de São Carlos, Universidade de São Paulo, Brazil \\ "Instituto de Física, Universidade Federal do Rio de Janeiro, Brazil \\ ${ }^{v}$ Núcleo de Formação de Professores - Universidade Federal de São Carlos, Brazil \\ ${ }^{w}$ Institute of Astronomy, BAS, Bulgaria \\ ${ }^{x}$ Institute for Nuclear Research and Nuclear Energy, BAS, Bulgaria \\ ${ }^{y}$ Astronomy Department of Faculty of Physics, Sofia University, Bulgaria \\ ${ }^{z}$ FESB - University of Split, Croatia \\ ${ }^{a}$ Rudjer Boskovic Institute, Croatia \\ ${ }^{a b}$ University of Rijeka, Physics Department, Croatia \\ ${ }^{a c}$ Institute of Physics of the Academy of Sciences of the Czech Republic, Czech Republic \\ ${ }^{a d}$ Charles University, Institute of Particle and Nuclear Physics, Czech Republic \\ ${ }^{a e}$ Aalto University, Finland \\ ${ }^{a f}$ University of Helsinki, Finland \\ ${ }^{a g}$ University of Oulu, Finland \\ ${ }^{a h}$ Tuorla Observatory, University of Turku, Finland \\ ${ }^{a i} A P C$, Univ Paris Diderot, CNRS/IN2P3, CEA/lrfu, Obs de Paris, Sorbonne Paris Cité, France, \\ France \\ ${ }^{a j} C E A / D S M / I R F U, C E A-S a c l a y$, France \\ ${ }^{a k}$ University of Bordeaux for the $C E N B G$, France \\ ${ }^{a l}$ Centre de Physique des Particules de Marseille (CPPM), Aix-Marseille Université, \\ CNRS/IN2P3, Marseille, France \\ ${ }^{a m}$ Institut de Planétologie et d'Astrophysique de Grenoble, INSU/CNRS, Université Joseph \\ Fourier, France \\ ${ }^{a n}$ Institut de Recherche en Astrophysique et Planétologie, France \\ ${ }^{a o}$ Laboratoire d'Annecy-le-Vieux de Physique des Particules, Université de Savoie, CNRS/IN2P3, \\ France \\ ${ }^{a p}$ Laboratoire Leprince-Ringuet, École Polytechnique (UMR 7638, CNRS), France \\ ${ }^{a q}$ LPNHE, University of Pierre et Marie Curie, Paris 6, University of Denis Diderot, Paris 7, \\ CNRS/IN2P3, France \\ ${ }^{a r}$ Laboratoire Univers et Particules de Montpellier, Université Montpellier 2, CNRS/IN2P3, \\ France
}




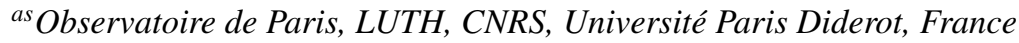 \\ ${ }^{a t}$ Deutsches Elektronen-Synchrotron, Germany \\ ${ }^{a u}$ Institut für Astronomie und Astrophysik, Universität Tübingen, Germany \\ ${ }^{a v}$ Landessternwarte, Universität Heidelberg, Germany \\ ${ }^{a w}$ Max-Planck-Institut für Kernphysik, Germany \\ ${ }^{a x}$ Max-Planck-Institut für Physik, Germany \\ ${ }^{a y}$ Department of Physics, TU Dortmund University, Germany \\ ${ }^{a z}$ Department of Physics, Humboldt University Berlin, Germany \\ ${ }^{b a}$ Institut für Theoretische Physik, Lehrstuhl IV: Weltraum- und Astrophysik, Ruhr-Universität \\ Bochum, Germany \\ ${ }^{b b}$ Universität Erlangen-Nürnberg, Physikalisches Institut, Germany \\ ${ }^{b c}$ Universität Hamburg, Institut für Experimentalphysik, Germany \\ ${ }^{b d}$ Institut für Physik and Astronomie, Universität Potsdam, Germany \\ ${ }^{b e}$ Institute for Theoretical Physics and Astrophysics, Universität Würzburg, Germany \\ ${ }^{b f}$ National Technical University of Athens, Department of Physics, Greece \\ ${ }^{b g}$ Faculty of Physics, National and Kapodestrian University of Athens, Greece \\ ${ }^{b h}$ School of Physics, Aristotle University, Thessaloniki, Greece

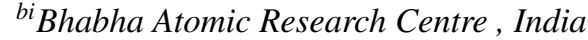 \\ ${ }^{b j}$ Saha Institute of Nuclear Physics, India \\ ${ }^{b k}$ Tata Institute of Fundamental Research, India \\ ${ }^{b l}$ Dublin Institute for Advanced Studies, Ireland \\ ${ }^{b m}$ University College Dublin, Ireland \\ ${ }^{b n}$ Department of Natural Sciences, The Open University of Israel, Israel \\ ${ }^{b o}$ IAPS Istituto di Astrofisica e Planetologia spaziali, Italy \\ ${ }^{b p}$ Istituto di Astrofisica Spaziale e Fisica Cosmica-Bologna, Italy \\ ${ }^{b q}$ Istituto di Astrofisica Spaziale e Fisica Cosmica, Italy \\ ${ }^{b r}$ INAF - Istituto di Astrofisica Spaziale e Fisica Cosmica di Palermo, Italy \\ ${ }^{b s}$ INFN Sezione di Bari, Italy \\ ${ }^{b t}$ INFN, Sezione di Como / Milano Bicocca, Italy \\ ${ }^{b u}$ INFN Sezione di Napoli, Italy \\ ${ }^{b v}$ INFN Sezione di Perugia, Italy \\ ${ }^{b w}$ INFN Sezione di Pisa, Italy \\ ${ }^{b x}$ INFN Sezione di Roma La Sapienza, Italy

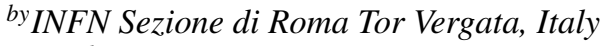 \\ ${ }^{b z}$ INFN Sezione di Torino, Italy \\ ${ }^{c a}$ Institute of Advanced Study of Pavia, Italy \\ ${ }^{c b}$ Osservatorio Astrofisico di Arcetri, Italy \\ ${ }^{c c}$ INAF - Osservatorio Astronomico di Bologna, Italy \\ ${ }^{c d}$ INAF - Osservatorio Astronomico di Brera, Italy \\ ${ }^{c e}$ INAF - Osservatorio Astrofisico di Catania, Italy \\ ${ }^{c f}$ INAF - Osservatorio Astronomico di Padova, Italy \\ ${ }^{c g}$ INAF - Osservatorio Astronomico di Roma, Italy \\ ${ }^{c h}$ Osservatorio Astronomico di Trieste and INFN Sezione di Trieste, Italy

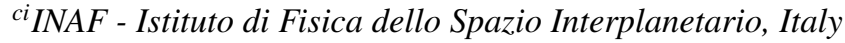 \\ ${ }^{c j}$ Project Department, INAF, Italy
}




\author{
${ }^{c k}$ INAF - Telescopio Nazionale Galileo, Italy \\ ${ }^{c l}$ Dipartimento di Fisica - Universitá degli Studi di Padova, Italy \\ ${ }^{\mathrm{cm}}$ University of Udine and INFN Sezione di Trieste, Italy \\ ${ }^{c n}$ Hiroshima Astrophysical Science Center, Hiroshima University, Japan \\ ${ }^{c o}$ Institute of Space and Astronautical Science, JAXA, Japan \\ ${ }^{c p}$ Institute of Particle and Nuclear Studies, KEK (High Energy Accelerator Research \\ Organization), Japan \\ ${ }^{c q}$ Department of Physics, Kyoto University, Japan \\ ${ }^{c r}$ Riken, Institute of Physical and Chemical Research, Japan \\ ${ }^{c s}$ Interactive Research Center of Science, Tokyo Institute of Technology, Japan \\ ${ }^{c t}$ Department of Physics and Mathematics, Aoyama Gakuin University, Japan \\ ${ }^{c u}$ Department of Physical Science, Hiroshima University, Japan \\ ${ }^{c v}$ Faculty of Science, Ibaraki University, Japan \\ ${ }^{c w}$ Dept. of Physics, Kinki University, Japan \\ ${ }^{c x}$ School of Allied Health Sciences, Kitasato University, Japan \\ ${ }^{c y}$ Department of Physics, Konan University, Japan \\ ${ }^{c z}$ Kumamoto University, Japan \\ ${ }^{d a}$ Department of Applied Physics, University of Miyazaki, Japan \\ ${ }^{d b}$ Department of Physics and Astrophysics, Nagoya University, Japan \\ ${ }^{d c}$ Kobayashi-Maskawa Institute (KMI) for the Origin of Particles and the Universe, Nagoya \\ University, Japan \\ ${ }^{d d}$ Solar-Terrestrial Environment Laboratory, Nagoya University, Japan \\ ${ }^{d e}$ Department of Earth and Space Science, Graduate School of Science, Osaka University, Japan \\ ${ }^{d f}$ Department of Physics, Rikkyo University, Japan \\ ${ }^{d g}$ Graduate School of Science and Engineering, Saitama University, Japan \\ ${ }^{d h}$ Department of Physics, Tokai University, Japan \\ ${ }^{d i}$ Tokai University Hospital, Japan \\ ${ }^{d j}$ Institute of Socio-Arts and Sciences, University of Tokushima, Japan \\ ${ }^{d k}$ Department of Astronomy, University of Tokyo, Japan \\ ${ }^{d l}$ Institute for Cosmic Ray Research, University of Tokyo, Japan \\ ${ }^{d m}$ Department of Physics, Graduate School of Science, University of Tokyo, Japan \\ ${ }^{d n}$ Faculty of Science and Engineering, Waseda University, Japan \\ ${ }^{d o}$ Department of Physics, Yamagata University, Japan \\ ${ }^{d p}$ Faculty of Management Information, Yamanashi-Gakuin University, Japan \\ ${ }^{d q}$ Universidad Nacional Autónoma de México, México, Mexico \\ ${ }^{d r}$ University of Namibia, Department of Physics, Namibia \\ ${ }^{d s}$ Radboud University Nijmegen, Netherlands \\ ${ }^{d t}$ Astronomical Institute Anton Pannekoek, University of Amsterdam, Netherlands \\ ${ }^{d u}$ Department of Physics and Technology, University of Bergen, Norway \\ ${ }^{d v}$ Faculty of Computer Science, Electronics and Telecommunications, AGH University of Science \\ and Technology, Kraków, Poland \\ ${ }^{d w}$ Academic Computer Centre CYFRONET AGH, Poland \\ ${ }^{d x}$ The Henryk Niewodniczański Institute of Nuclear Physics, Polish Academy of Sciences, Poland \\ ${ }^{d y}$ Copernicus Astronomical Center, Polish Academy of Sciences, Poland \\ ${ }^{d z}$ Space Research Centre, Polish Academy of Sciences, Poland
}


${ }^{e a}$ Toruń Centre for Astronomy, Nicolaus Copernicus University, Poland

${ }^{e b}$ Faculty of Physics, Astronomy and Applied Computer Science, Jagiellonian University, Poland

${ }^{e c}$ Faculty of Physics and Applied Computer Science, University of Lódź, Poland

${ }^{\text {ed }}$ Faculty of Physics, University of Warsaw, Poland

${ }^{e}$ Laboratory for Astroparticle Physics, University of Nova Gorica, Slovenia

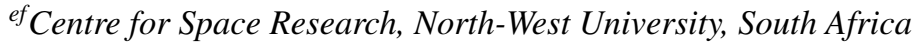

${ }^{e g}$ University of Johannesburg, Department of Physics, South Africa

${ }^{\text {eh }}$ University of the Witwatersrand, South Africa

${ }^{\text {ei }}$ University of the Free State, South Africa

${ }^{\text {ej CIEMAT, Spain }}$

${ }^{e k}$ Instituto de Astrofísica de Canarias, Spain

${ }^{e l}$ Departament d'Astronomia i Meteorologia, Institut de Ciències del Cosmos, Universitat de Barcelona, Spain

${ }^{\text {em }}$ Institut de Ciències de l'Espai (IEEC-CSIC) and Institució Catalana de Recerca I Estudis

Avançats (ICREA), Spain

en Institut de Física d'Altes Energies, Spain

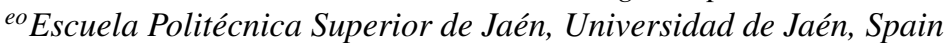

${ }^{e p}$ Unitat de Física de les Radiacions, Departament de Física, and CERES-IEEC, Universitat

Autònoma de Barcelona, E-08193 Bellaterra, Spain, Spain

${ }^{e q}$ Grupo de Altas Energías, Universidad Complutense de Madrid., Spain

${ }^{e r}$ Grupo de Electronica, Universidad Complutense de Madrid, Spain

${ }^{\text {es }}$ Lund Observatory, Lund University, Sweden

${ }^{e t}$ Oskar Klein Centre, Department of Physics, Royal Institute of Techology (KTH), Sweden

${ }^{e u}$ Linnaeus University, Sweden

ev Stockholm University, Sweden

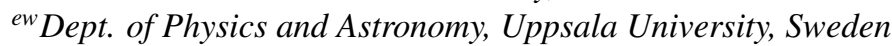

${ }^{e x}$ Laboratory for High Energy Physics, École Polytechnique Fédérale, Switzerland

${ }^{e y}$ ETH Zurich, Institute for Particle Physics, Switzerland

${ }^{e z}$ University of Geneva - Département de physique nucléaire et corpusculaire, Switzerland

${ }^{f a}$ ISDC Data Centre for Astrophysics, Observatory of Geneva, University of Geneva, Switzerland

${ }^{f b}$ Physik-Institut, Universität Zürich, Switzerland

${ }^{f c}$ King's College London, United Kingdom

${ }^{f d}$ STFC Rutherford Appleton Laboratory, United Kingdom

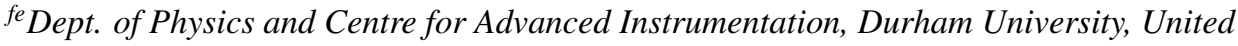

Kingdom

${ }^{f f}$ School of Physics and Astronomy, University of Edinburgh, United Kingdom

${ }^{f g}$ Centre for Astrophysics Research, Science and Technology Research Institute, University of Hertfordshire, United Kingdom

${ }^{\text {fh }}$ Dept. of Physics and Astronomy, University of Leicester, United Kingdom

${ }^{f}$ The Astrophysics Research Institute, Liverpool John Moores University, United Kingdom

${ }^{f j}$ School of Physics and Astronomy, University of Nottingham, United Kingdom

${ }^{f k}$ University of Oxford, Department of Physics, United Kingdom

${ }^{f l}$ Department of Physics and Astronomy, University of Sheffield, United Kingdom

${ }^{f m}$ School of Physics and Astronomy, University of Southampton, United Kingdom

${ }^{\text {fn }}$ School of Physics and Astronomy, University of Leeds, United Kingdom 


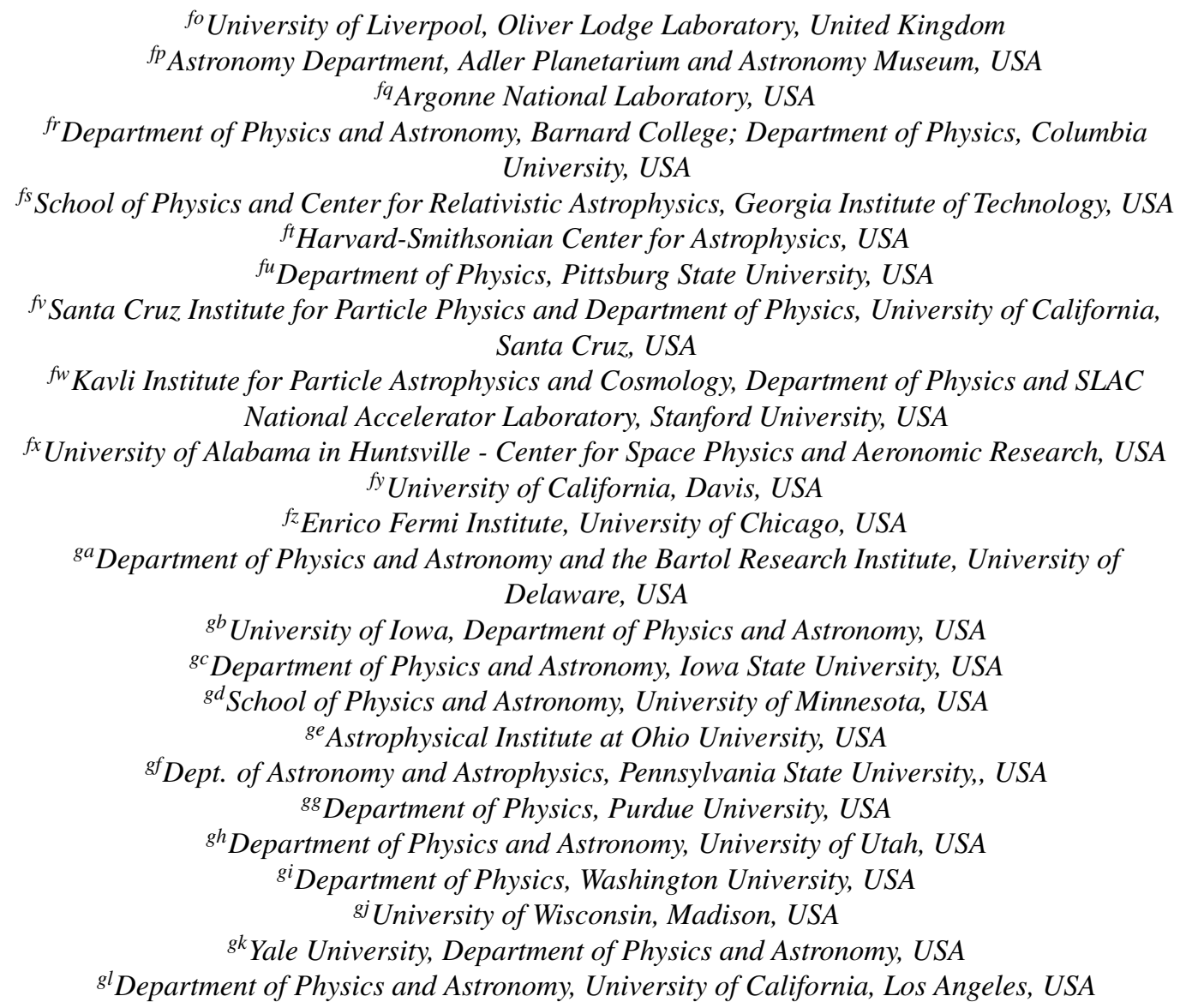

\begin{abstract}
Supernova remnants (SNRs) are among the most important targets for $\gamma$-ray observatories. Being prominent non-thermal sources, they are very likely responsible for the acceleration of the bulk of Galactic cosmic rays (CRs). To firmly establish the SNR paradigm for the origin of cosmic rays, it should be confirmed that protons are indeed accelerated in, and released from, SNRs with the appropriate flux and spectrum. This can be done by detailed theoretical models which account for microphysics of acceleration and various radiation processes of hadrons and leptons. The current generation of Cherenkov telescopes has insufficient sensitivity to constrain theoretical models. A new facility, the Cherenkov Telescope Array (CTA), will have superior capabilities and may finally resolve this long standing
\end{abstract}


issue of high-energy astrophysics. We want to assess the capabilities of CTA to reveal the physics of various types of SNRs in the initial 2000 years of their evolution. During this time, the efficiency to accelerate cosmic rays is highest. We perform time-dependent simulations of the hydrodynamics, the magnetic fields, the cosmic-ray acceleration, and the non-thermal emission for type Ia, Ic and IIP SNRs. We calculate the CTA response to the $\gamma$-ray emission from these SNRs for various ages and distances, and we perform a realistic analysis of the simulated data. We derive distance limits for the detectability and resolvability of these SNR types at several ages. We test the ability of CTA to reconstruct their morphological and spectral parameters as a function of their distance. Finally, we estimate how well CTA data will constrain the theoretical models.

Keywords: acceleration of particles, gamma rays: general, ISM: supernova remnants, radiation mechanisms: non-termal

\section{Introduction}

SNRs are the main candidate sites for the acceleration of cosmic rays (CRs) in the Galaxy (Ginzburg \& Syrovatsky, 1961). They satisfy the energy requirements for re-filling the Galaxy with relativistic particles, and the theory of diffusive shock acceleration can describe the acceleration of cosmic rays to $\mathrm{PeV}$ energies at SNR shocks. SNRs are powerful sources of non-thermal radiation in the radio, $\mathrm{X}$-ray, and $\gamma$-ray bands, indicating the existence of high-energy cosmic-ray electrons in the remnants. Protons are much less efficient emitters than electrons and therefore harder to identify. However, they must undoubtebly be accelerated along with electrons in order to provide magnetic turbulence responsible for particle scattering upstream of the SNR shock. Relativistic protons can produce neutral pions in collisions with matter (Stecker, 1973). The pions decay and produce $\gamma$-rays with energies approximately an order of magnitude lower than that of the parent protons. The signatures of neutral pion-decay in SNRs were observed by Fermi-LAT (Ackermann et al., 2013). Therefore, observations at $\gamma$-ray energies are a powerful tool for understanding the processes of cosmic-ray (nuclei) acceleration and propagation in the vicinity of strong SNR shocks. The hadronic

\footnotetext{
*corresponding author

** corresponding author

Email addresses: maxim.shayduk@desy.de (M. Shayduk), igor.telezhinsky@desy.de (I. Telezhinsky)
} 


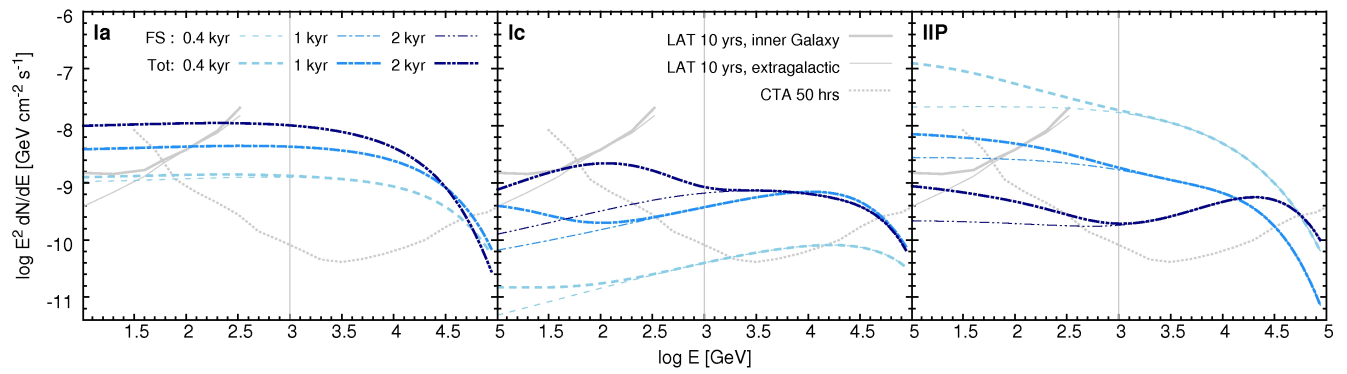

Figure 1: Theoretical $\gamma$-ray emission spectra due to pion-decay and inverse Compton emission for type-Ia (left), type-Ic (center) and type-IIP (right) SNRs, ages of 400, 1000, 2000 years, and a distance of $1.5 \mathrm{kpc}$. The acronym "kyr" stands for a kiloyear. The "FS" label denotes spectra due to the acceleration in the forward shock (FS) only and the label "Tot" denotes the total emission spectra due to particle acceleration at both forward and reverse shocks. The Fermi-LAT sensitivities are given for 10 years exposure time, Galactic and extragalactic background levels (details can be found in Funk et al., 2013), compared to the sensitivity of CTA for 50 hours exposure time. For all sensitivities the detection significance of 5 standard deviations is required.

$\gamma$-rays may be mixed with inverse Compton (IC) emission of the electrons. Explanation of $\gamma$-ray SNR spectra requires sophisticated theoretical modelings accounting for different contributions of hadrons and leptons to the total emission. The models must be guided by precise measurements of the energy spectra and morphology. A new generation facility, the Cherenkov Telescope Array (Actis et al., 2011; Acharya et al., 2013) will provide these measurements, complementing the Fermi-LAT data at energies above $50 \mathrm{GeV}$ (see sensitivity curves shown in Fig. 1).

A preliminary assessment of the capabilities of CTA to detect and resolve sources with properties similar to currently $\gamma$-ray detected SNRs has been accomplished earlier (Renaud \& CTA Consortium, 2011; Acero et al., 2013). However, in these studies the known $\gamma$-ray SNRs RX J1713.7-3946 and Vela Junior were adapted as representatives of the general SNR population. These two SNRs are possibly special cases and not as generic as assumed there. In contrast, the study presented in this paper uses a general and realistic model of SNR physics. Moreover, it takes into account the most interesting early epochs of SNR evolution, when CRs may reach PeV energies and their emission may show important spectral features and morphology.

The goal of this paper is to test the potential of CTA to measure various aspects 
of physics of young SNRs, including both spectral and morphological properties of sources. For this we use detailed theoretical models of SNRs that are built on time-dependent simulations of the hydrodynamical and magnetic field evolution, as well as the cosmic-ray acceleration at forward and reverse shocks with re-acceleration at secondary reflected shock structures (Telezhinsky et al., 2012a, 2013). We compute the broadband emission from leptonic and hadronic populations and produce a set of models for the remnants of various Supernova (SN) types, namely type Ia, type Ic, and type IIP. Furthermore, we produce realistic high-resolution astrophysical background maps using 3-dimentional (3-D) Galactic plane gas distribution measurements (Pohl et al., 2008). The various SNR models and the astrophysical backgrounds are used in the form of intensity maps as inputs to detailed simulations of the CTA response, taking into account the off-axis performance of the instrument. We then analyze the simulated data and reconstruct the spectral and morphological parameters. We attempt to derive from the data the basic observables of the sources, such as detection and resolution significance, spectral indices, and cut-off energies. We compare the derived values to the theoretical ones and set limits on the feasibility of reconstructing the parameters of the source model and constraining theoretical models of the sources.

\section{Theoretical modeling}

Our theoretical modeling is described in a series of earlier papers (Telezhinsky et al., 2012a,b, 2013) that are based on a kinetic approach and take into account the complicated hydrodynamics of SNR evolution in an arbitrary circumstellar medium (CSM). We numerically solve the transport equation of cosmic rays in the test-particle approximation and a spherically-symmetric geometry using the flow parameters derived from high-resolution hydrodynamical simulations. The magnetic field (MF) amplification is not considered here. We assume that the amplitudes of turbulent and regular components of the MF are roughly equal. The evolution of the regular component of the MF is followed by solving the induction equation in the plasma-flow profiles. The ambient medium MF values are different for considered SNR types (e.g., $B_{0}=5 \mu \mathbf{G}$ in CSM of type-Ia SNR, and $B_{0}$ changes from a few dozens to a few $\mu \mathrm{G}$ in the CSM of core-collapse SNRs). We consider particle acceleration by at least two main shocks in the SNR, the forward shock that propagates in the CSM and the reverse shock that propagates in the stellar ejecta. So particles are injected at these shocks. Additionally, all secondary reflected shock structures that may be present in the young SNR are included and participate in particle re-acceleration. The 
injection of particles is treated according to thermal-leakage model of Blasi et al. (2005). We adjust the injection efficiency so that the CR pressure limit (10\% of shock ram pressure) imposed by test-particle approximation is not violated. Note that the injection efficiency in this case does not affect the resulting emissivities because for denser shocks the smaller injection efficiency is taken (e.g., $5 \times 10^{-7}$ for type-Ia, $5 \times 10^{-6}$ for type-Ic and $5 \times 10^{-8}$ for type-IIP SNR). The number of injected protons and electrons is assumed to be equal. The electron to proton ratio, $K_{e p}$, is usually the ratio of particle intensities at some energy. In our case it is roughly the mass ratio of electrons to protons.

The models used in the current study account for complicated hydrodynamics of SNR evolution and at the same time all basic physics of particle acceleration. Our models can be considered rather conservative. We are aware of existing other approaches to theoretical modeling of SNRs that include the effects of non-linear CR acceleration (Berezhko \& Völk, 1997) in strongly amplified MFs (Zirakashvili \& Ptuskin, 2008; Ptuskin et al., 2010; Zirakashvili \& Ptuskin, 2011; Caprioli et al., 2009; Kang et al., 2013; Bykov et al., 2014), advanced hydrodynamics (Schure et al., 2010) or altogether (Lee et al., 2012; Ferrand et al., 2014). To our knowledge there is no absolutely assumption free approach and each of the approaches has focused on and advanced certain physics aspects at the cost of simplifying or neglecting the other (see e.g., Caprioli et al. (2010) for comparisons of some approaches). The major effect of non-linear acceleration on cosmic ray spectrum is so called concavity - soft low-energy and hard high-energy parts of particle distribution. To some extent, the similar effect can be produced by particle acceleration at the reverse shock (see Fig. 1, type-IIP SNR spectrum at the age of 2000 years). Unfortunately, neither of the effects is established by very high-energy (VHE) observations, though there are hints for both effects at other wavelengths (radio, X-rays). The MF amplification would affect the maximum energy of particles and hence the emission cut-off region by moving it to higher energies. It can also decrease leptonic contribution to VHE band. If MF is amplified, one needs less electrons (smaller $K_{e p}$ ) to explain X-ray intensity and therefore the leptonic emission in VHE band should be also weaker. Thus we consider upper limits on leptonic component of VHE spectra.

To cover a wide range of spectral parameters, luminosities, and morphological types of SNRs, we studied the remnants of various explosion types such as thermonuclear explosions of white dwarfs (type Ia) and core-collapse of massive stars (type Ic and type IIP). These types cover $\simeq 90 \%$ of known explosions (Smartt et al., 2009; Ptuskin et al., 2010). It worth mentioning that computations of 
particle spectra for these types were also done by Ptuskin et al. (2010, 2013). They did not consider any radiation from the SNRs. They used much simpler hydrodynamics but accounted for highly modified shocks. They arrived at different particle spectra, which is not surprising given different assumptions and aims pursued in their work. The trends reported here should be generic if DSA efficiency stays within $10 \%$. Note however, that owing to inclusion of acceleration at the reverse shock some of our spectra resemble ones obtained within non-linear DSA. Therefore, CTA performances derived here for these spectra should be also applicable to similar non-linearly modified spectra.

Blast waves of type Ia SNe normally propagate in uniform CSM with densities typical for the interstellar medium (ISM) of the Galaxy. The shapes of their spectra weakly depend on time already after a few hundred years of evolution (Telezhinsky et al., 2012a) and can be described by a power-law of index $s \simeq 2$ with an exponential cutoff at high energies (for the time evolution of their $\gamma$-ray spectra see Fig 1, left panel). The free-expansion stage of these SNRs ends rather quickly, and roughly after 1000 years their evolution can be perfectly described by self-similar Sedov-Taylor solutions. The best examples of type-Ia SNRs are Tycho, SN 1006, and Kepler. We have shown (Telezhinsky et al., 2013) that contrary to type-Ia SNRs, the emission spectra of core-collapse SNRs strongly depend on the environment of the SN explosion, through which the blast wave propagates. This environment is shaped by the winds of the SN progenitor star during its late evolution. Even a few thousand years after explosion, core-collapse SNRs may not reach the Sedov-Taylor stage. The reverse shock, as well as several secondary reflected shocks inside the outer blast wave (the forward shock), may be still strong enough to accelerate and re-accelerate particles. This modifies the particle distributions and the resulting emission spectra of core-collapse SNRs. Their spectra are no longer simple power laws with exponential cutoffs, but rather complex with spectral index changing with energy and time (see Fig 1, middle and right panels). Moreover, leptonic and hadronic emission contributes at different levels in different regions of the SNR at different ages, as opposed to type-Ia SNRs where the emission is predominantly of hadronic origin. The spread of model parameters of core-collapse SNRs is much wider than that of type Ia SNRs, owing to the variety of progenitor star masses leading to different mass-loss rates, wind velocities and durations of evolutionary phases the progenitor stars go through. Not only can distinct types of SN explosions be distinguished, the subsequent evolution of their remnants also display substantial variations. Here we limit ourselves to the most frequent types with rather generic parameters representative of 
these cases. Namely, type Ic, which are explosions of Wolf-Rayet (WR) stars, and type IIP, which are explosions of red supergiant (RSG) stars. WR and RSG have considerably different wind velocities, leading to a large variation in the ambient medium into which the SNR expands. Many SNe, even those of other types, will fall somewhere close to or in between these types. For instance, Cas A is classified as a type IIb SN based on spectra obtained from light echoes. However, its evolution in a RSG wind (Chevalier \& Oishi, 2003), within which it is probably currently expanding, is quite similar to the evolution in a wind medium for a type IIP SN. Thus, with our models we encompass the spectra and evolution of many different types of young SNRs. We stop our simulations at 2000 years because for our parameters type-Ia SNR is already long in Sedov stage whereas core-collapse SNRs start loosing in luminosity while their forward shocks may approach the wind-blown shells of progenitor stars and illuminate them with escaping CRs (Telezhinsky et al., 2012b). Here we would like to focus on the emission from SNRs only. The inputs to our simulations and data analysis are described in the following subsections.

\subsection{Thermonuclear explosion: type-Ia SNRs.}

To study the evolution of young type-Ia SNe, we need at least two parameters. A density profile of the stellar ejecta and that of the CSM. Since the progenitor star is a low-mass star and does not undergo significant mass loss, the CSM is assumed to be constant-density ISM. The ejecta density structure is more uncertain. However, by comparing spherically-symmetric models of type-Ia SN explosions, it was found (Dwarkadas \& Chevalier, 1998) that the ejecta structure can be best represented by an exponential density profile, which we therefore use in our simulations. The initial conditions depend on three parameters: (i) the energy of the ejected material, which we take as the canonical $10^{51} \mathrm{erg}$, (ii) the mass of the ejecta, which we take as $1.4 \mathrm{M}_{\odot}$, and (iii) the number density of the CSM, which we take around $0.4 \mathrm{~cm}^{-3}$ similar to that in the vicinity of Tycho's SNR. The supersonic expansion of the $\mathrm{SN}$ ejecta into the ambient medium gives rise to a forward shock (FS) expanding into the CSM, and a reverse shock (RS) propagating through the ejecta. The two are separated by a contact discontinuity (CD), which separates the shocked ejecta from the shocked ambient medium. At the age of 1000 years we turn to Sedov-Taylor solutions since the contribution of the RS accelerated particles to the emission becomes negligible (Telezhinsky et al., 2012a). 


\subsection{Core-collapse explosions: type-Ic and type-IIP SNRs.}

Additional parameters are needed to model core-collapse SNRs because of their winds modifying the CSM. The progenitors of type-IIP SNe are RSG stars. As a massive star moves off the main sequence into the RSG stage, it grows considerably in size, the wind mass-loss rate increases to about $5 \times 10^{-5} M_{\odot} \mathrm{yr}^{-1}$ while the wind velocity drops to about $10 \mathrm{~km} \mathrm{~s}^{-1}$. This results in a new pressure equilibrium. The high density ( $\propto \dot{M} / v_{w}$ ) of the RSG wind leads to the formation of a wind region with a density almost four orders of magnitude above that of the main-sequence wind.

Progenitors of type-Ic SNe are WR stars, whose mass-loss rates are somewhat lower than those of RSGs. Their wind velocities are more than two orders of magnitude higher, leading to correspondingly lower wind densities. The high momentum of the winds pushes outwards onto the RSG shell, breaking it up in this process and mixing its material into the WR wind (Dwarkadas, 2007). This mixed material approaches the main-sequence shell, and eventually the system reaches an equilibrium situation that in many ways resembles a main-sequence star bubble.

In each case we assumed an ejecta mass of about $5 \mathrm{M}_{\odot}$ and an explosion energy of $10^{51} \mathrm{erg}$. The ejecta density is flat where the flow velocity is below a certain value, $u_{f l}$, and decreases as a power law with radius, $\rho_{e j} \propto r^{-9}$, where the flow velocity is above $u_{f l}$ (Chevalier \& Fransson, 1994; Dwarkadas, 2005). The interaction of the ejecta with the wind medium sets up a double-shock structure, consisting of a FS and RS separated by a CD.

The shock expansion in the wind medium is initially quite similar in both cases. The evolution changes and is no longer self-similar, once the FS reaches the end of the freely expanding wind region. In the RSG case (SN type IIP) one finds a huge drop in density beyond the wind region, whereas in the WR case (SN type Ic) one finds a termination shock and an increase in density by a factor of four. The interaction of the FS with the wind termination shock leads to a reflected shock that travels back into the ejecta in the case of type-Ic SNR. For type-IIP SNR, the steep drop leads to the formation of a complicated flow structure. These flow profiles are used to compute the acceleration and transport of particles at the shock fronts.

\subsection{Astrophysical background}

We constructed maps of the Galactic diffuse emission with pixel size $0.1^{\circ}$ for the region centered on the Galactic coordinates $\left(l=338^{\circ}, b=0^{\circ}\right)$, representing the

moderate emission intensity. As we are interested in the detectability of sources 

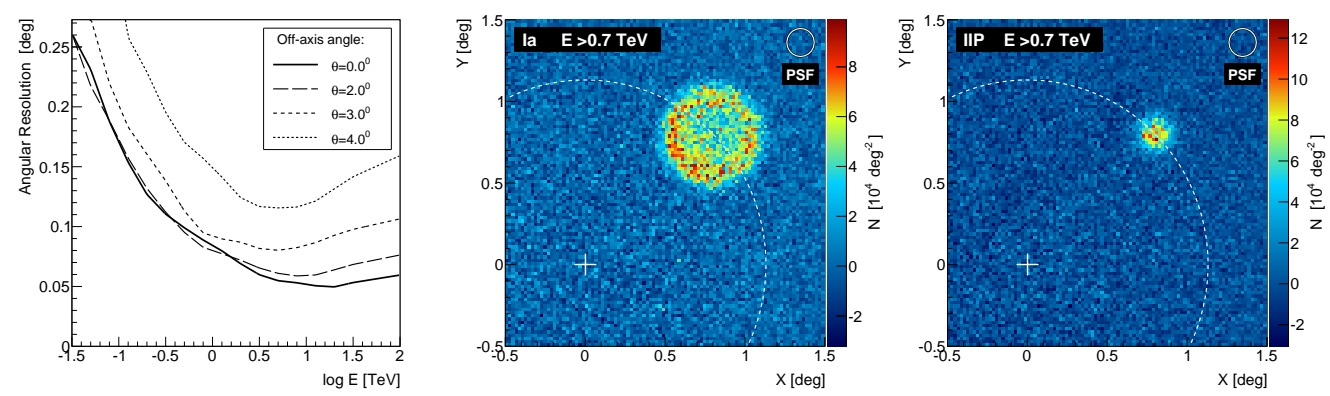

Figure 2: Left: Angular resolution curves $\Phi_{\text {AngRes }}(\theta=$ const,$E)$ of CTA for different off-axis angles $\theta$, evaluated from the full two-dimensional CTA response histogram $\Phi_{\text {AngRes }}(\theta, E)$. Center: Simulated count map of 2000 year-old type-Ia SNR at the distance of $1.5 \mathrm{kpc}$ for energies above $0.7 \mathrm{TeV}$ after residual background subtraction for an exposure time of 50 hours. The center of the instrument FoV is depicted with the white cross. The radius of the white dashed circle corresponds to the off-axis angle for reflected-region background estimation method. Right: Count map for a 1000 year-old type-IIP SNR at the distance of $1.5 \mathrm{kpc}$.

in a structured background, we ignore inverse Compton scattering and concentrate on hadronic emission. The gamma-ray emissivity is calculated by folding the gamma-ray production tables of Huang et al. (2007) with the locally observed (Menn et al., 2000; Adriani et al., 2011; Apel et al., 2012) cosmic-ray proton and helium spectra, which we approximate as $N_{\mathrm{H}}(E)=\left(4.2 \cdot 10^{-10} \mathrm{GeV}^{-1} \mathrm{~cm}^{-3}\right) E_{\mathrm{GeV}}^{-2.7}$ and $N_{\mathrm{He}}(E)=\left(1.6 \cdot 10^{-11} \mathrm{GeV}^{-1} \mathrm{~cm}^{-3}\right) E_{\mathrm{GeV}}^{-2.55}$, where $E_{\mathrm{GeV}}$ is the energy per nucleon in $\mathrm{GeV}$. Both spectra are assumed to steepen at $3 \mathrm{PeV}$ to an index of 3.2.

The column densities of gas are based on the CO survey of Dame et al. (2001) scaled with a conversion factor $X=4.6 \cdot 10^{20} \mathrm{H}-$ atoms cm${ }^{-2} \mathrm{~K}^{-1} \mathrm{~km}^{-1} \mathrm{~s}$. For atomic gas we use the LAB survey (Kalberla et al., 2005) and, where available, the Southern Galactic Plane Survey (SGPS) (McClure-Griffiths et al., 2005) with conversion factor $2.8 \cdot 10^{18} \mathrm{H}-$ atoms cm$~_{-2} \mathrm{~K}^{-1} \mathrm{~km}^{-1} \mathrm{~s}$. The spin temperature of diffuse atomic hydrogen is assumed to be high enough to render the radiation transport linear, except for small-scale hydrogen self-absorption in the high-resolution map of the SGPS which we corrected using the method of Gibson et al. (2005).

We ignore the possibility of confusion with other, possibly unresolved $\gamma$-ray sources in the field of view, which will expect to occur for some of the CTA observations of SNRs. 


\section{Simulating a CTA data set}

To take into account constraints arising from the analysis of the CTA data, we carefully simulated the event-processing steps, using response functions of the CTA instrument determined from detailed Monte-Carlo simulations (Bernlöhr et al., 2013). We focus on the performance of one possible realization of CTA, array layout $E$, described in Actis et al. (2011). This array comprises 4 large-size telescopes (LSTs), 23 mid-size telescopes (MSTs) and 32 small-size telescopes (SSTs) with reflector diameters of $23 \mathrm{~m}, 12 \mathrm{~m}$, and $7 \mathrm{~m}$, correspondingly. The layout covers an area of roughly $1 \mathrm{~km}^{2}$ and has a balanced sensitivity over a wide energy range from $30 \mathrm{GeV}$ to $300 \mathrm{TeV}$.

Our simulations start with generating $\gamma$-candidate events, since all detector response functions relevant for the presented data analysis are defined after the gamma/background separation procedure. Here, the term background stands for events from hadronic and electron-initiated air showers.

In our study we consider a non-uniform sensitivity of the instrument over the simulated field of view (FoV). All detector response functions $\Phi$ are functions of two arguments: off-axis angle $\theta$ and $\gamma$-candidate event energy $E$. We use the following notation: $\Phi_{\text {name }}(\theta, E)$, where the subscript "name" denotes the corresponding detector property, such as collection area - "CollArea", angular resolution "AngRes", energy resolution - "EnRes", reconstructed energy bias - "EnBias" and residual background rate after gamma/background separation cuts - "BgRate". All response functions are derived from CTA Monte-Carlo studies and are stored as 2-D histograms. An example of CTA response functions for different off-axis angles is shown in the left panel of Fig.2. The angular resolution, $\Phi_{\text {AngRes }}(\theta, E)$, is defined as the radius within which $80 \%$ of the total events from a point source are contained. Below we refer to this quantity as the point-spread function (PSF).

As primary input for the simulation of a CTA data set we use the theoretical $\gamma$ ray intensity maps, placed within the instrument FoV with a certain off-axis angle to simulate the observations in wobble mode (Daum et al., 1997). This observation mode allows using the same data set for both the signal and the background estimation, which reduces uncertainties arising from inhomogeneous telescope and camera performance. For every source under consideration the theoretical sky maps are produced as a function of energy for the energy range from $10 \mathrm{GeV}$ to $100 \mathrm{TeV}$ with energy steps of $\Delta E / E=0.1$. These intensity maps are convolved with the CTA collection area $\Phi_{\text {CollArea }}(\theta, E)$ to obtain the expected number of counts for a predefined observation time of 50 hours. The corresponding $\gamma$-ray count maps are derived assuming Poisson statistics. Every entry from this se- 

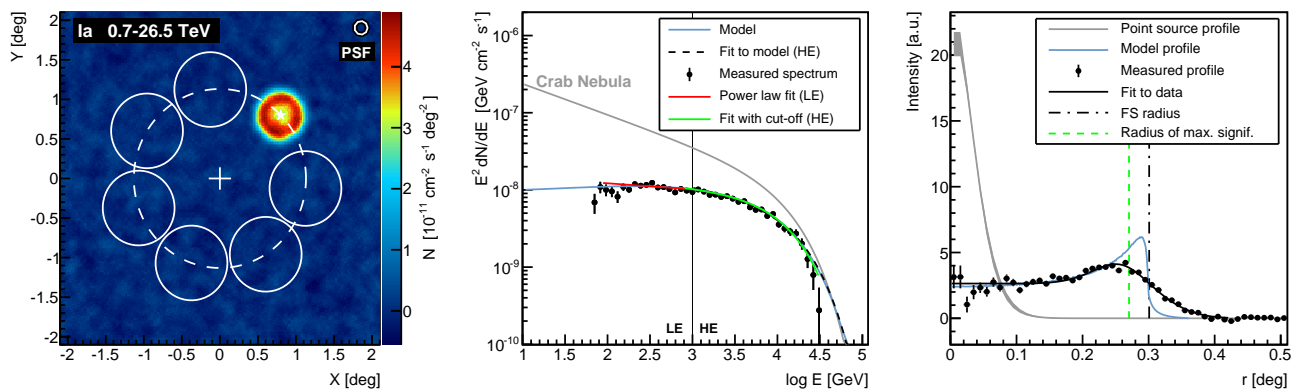

Figure 3: Left: Gamma-ray image of a 2000 year-old type-Ia SNR at the distance of $1.5 \mathrm{kpc}$ for energies above $0.7 \mathrm{TeV}$ smoothed with a cylindrical kernel with the radius of $0.07^{\circ}$. The exposure time is 50 hours. The center of the FoV is depicted with the white cross and the radius of the white dashed circle corresponds to the off-axis angle of reflected-region method positions (white circles). Center: Reconstructed energy spectrum with fit functions for different energy ranges as discussed in the text. Right: Reconstructed intensity profile for energies above $0.7 \mathrm{TeV}$ (black circles) with the profile fit defined by Eq. 3 (black curve). The grey curve with shaded error band represents the profile of a point source. The error band corresponds to a systematic uncertainty of $5 \%$ in the PSF determination.

quence of "true" $\gamma$-events is processed to have a "measured" direction and energy by convolving it with the CTA angular resolution $\Phi_{\text {AngRes }}(\theta, E)$ and energy migration matrix. We approximated the energy migration matrix as a superposition of two operations: an energy spread according to the energy resolution $\Phi_{\text {EnRes }}(\theta, E)$ and a bias on the event energy following the response function $\Phi_{\text {EnBias }}(\theta, E)$. The source map processing, as described above, is also performed for the diffuse astrophysical $\gamma$-ray background sky maps.

Finally, background $\gamma$-candidate events are added according to the residual background rate distribution $\Phi_{\text {BgRate }}(\theta, E)$, which comprises $\gamma$-like events from both cosmic-ray hadrons and electrons. In contrast to other response functions, here the second argument $E$ denotes the reconstructed energy of the $\gamma$-like event, not the true energy of the primary particle. Thus the energy migration matrix is not applied for these events. Examples of simulated sky maps are depicted on the central and right panels of Fig 2. For an extended source (central panel) the axial symmetry of the source is clearly modulated by the non-uniform instrument sensitivity. 


\section{Data analysis}

The simulated CTA data set is a sequence of $\gamma$-candidate events with estimated arrival direction and $\gamma$-ray energy. The final analysis of these data will determine the limits of the CTA instrument performance, including the source-detection potential, resolution of extended sources, measurable energy range and the feasibility of source-morphology studies.

We assume that the position of the source center is known from observations at other wavelengths (e.g. from radio or X-ray observations). In our simulations the source is placed in the instrument FoV with an offset with respect to the FoV center, thus the same data set can be used for the background extraction and no specific off-source data are required. The background subtraction is performed with two methods: background estimation using the reflected-region method (Daum et al., 1997; Bretz et al., 2005) and the so-called ring-background method (Berge et al., 2007), both assuming axial symmetry of the residual CR background with respect to the center of the FoV. Here we should note that the astrophysical diffuse $\gamma$-ray background does not necessarily follow this axial symmetry and can potentially lead to systematic uncertainties in the background subtraction procedure for both the reflected-region and the ring-background methods, especially for instruments with improved $\gamma$-ray sensitivity like the CTA facility. The reflected-region option is used for the reconstruction of the $\gamma$-ray energy spectrum, integrated over a certain region of interest, usually the total area occupied by the source. Naturally, the ring-background method with fine radial resolution $\left(0.03^{\circ}\right)$ is used for source morphology studies. In this approach, after residual-background subtraction, the raw $\gamma$-ray count maps are obtained for predefined energy intervals. The maps are converted to sky intensity maps (in units of $\mathrm{GeV}^{-1} \mathrm{~cm}^{-1} \mathrm{~s}^{-1} \mathrm{deg}^{-2}$ ), using the collection area response function $\Phi_{\text {CollArea }}(\theta, E)$ and the corresponding flux profiles are reconstructed in bins of energy (see Fig. 3).

\subsection{Detectability of sources}

We define the limit of source detectability as the statistical significance of $5 \sigma$, where $\sigma$ denotes the standard deviation. Such high value is usually required in operating imaging Cherenkov telescope experiments to avoid spurious detections due to systematic errors and fluctuations, arising, e.g., from uncertain hardware performance or limits in the modeling of the measurement process with MonteCarlo simulations.

The statistical significance of detection, $S_{\text {det }}$, is calculated according to the expression derived in Li \& Ma (1983) (Eq. 17) with the ratio of on-source time 
to off-source time, $\alpha=0.2$. The value of the significance, $S_{\text {det }}$, depends on the sky area assigned to the source. The extension and the complicated morphology of the source in $\gamma$-rays are not known a priori. We still assume axial symmetry of the source, so that the detection significance becomes a function of the radius of the region of interest.

The on-source counts, $N_{\text {on }}(r, E)$, and the off-source counts, $N_{\text {off }}(r, E)$, are extracted as cumulative values for both region of interest (radius $r$ ) and energy $E$. The spatial integration over the region of interest starts at the source center, and the integration over energies is performed from high towards low energies. Then, the corresponding cumulative detection significance is calculated and the maximum value of $S_{\text {det }}$ for a certain radius $r=R_{\mathrm{MS}}$ and energy $E=E_{\mathrm{MS}}$ is determined. In this manner, the eventual complex source morphology is taken into account, and the extent of the region of interest does not need to be defined a priori. However, the described procedure introduces a trial factor. To correct for this trial factor, we obtained the full probability density distribution of $S_{\text {det }}$ from simulations of sky maps with no $\gamma$-ray counts from SNRs. Moreover, two additional conditions are applied in the derivation of the detection significance. The first condition is that the excess to background count ratio be $\left(N_{\text {on }}-N_{\text {off }}\right) / N_{\text {off }}>\eta$, which constrains the analysis energy threshold. The second condition defined as $N_{\text {on }}-N_{\text {off }}>\beta$ limits the instrument dynamic range at high energies. These conditions are widely used in current Cherenkov-telescope experiments. We have set $\eta=0.05$ and $\beta=10$ for logarithmic binning in energy with five bins per decade.

\subsection{Resolvability of sources}

As a source-resolvability criterion, we have chosen the point-source hypothesis test. The profile of a point-source (see Fig. 3, right panel) is fully determined by the instrument PSF. One should keep in mind that the PSF depends on the $\gamma$ ray energy, thus it is necessary to build the point-source profile within the same energy range and with the same energy spectrum as the source under consideration. We assume that the systematic uncertainty of the instrument PSF parameter due to telescope pointing errors and the finite precision of PSF measurements is $5 \%$. This constrains the resolvability of compact but substantially bright sources in cases when statistical fluctuations become comparable or smaller than the introduced systematics.

In order to test the point source hypothesis, we extract the observed source profile residuals (with respect to the profile of a point source) and calculate the corresponding $\chi^{2}$ value. We chose a point-source hypothesis rejection level of $S_{\text {res }}>3 \sigma$. For the final resolvability analysis under conditions of low statistics, 
the flux profiles with initial fine binning of $0.02 \mathrm{deg} / \mathrm{bin}$ are re-binned by an automatic procedure to maintain $S_{\text {bin }}>2.5 \sigma$, where $S_{\text {bin }}$ is the average significance in one profile bin.

The energy range for the final profile is chosen exploiting the detectabilityanalysis results. The energy of maximum significance, $E_{\mathrm{MS}}$, is used as the lowenergy limit of the profile calculation. The high-energy limit is determined by the excess counts statistics, $N_{\text {on }}-N_{\text {off }}>10$.

\subsection{Spectral reconstruction}

As for the detectability analysis, the energy range available for spectral studies is constrained by the analysis threshold, $E_{\min }$, and the statistics of excess counts determining the upper boundary, $E_{\max }$, of the spectral range. After background subtraction according to the reflected-region method, the excess counts are extracted for every energy interval and converted to a $\gamma$-ray flux using the angledependent collection area, $\Phi_{\text {CollArea }}(\theta, E)$. We also add a systematic uncertainty of $5 \%$ to the reconstructed flux values.

To cover the full spectral variety with simple fitting functions, we split the measurable energy range into two energy bands: the low-energy band (LE), where the contribution of the particles accelerated at the RS to the emission may be significant (see Fig. 1), and the high-energy band (HE) dominated by the emission of particles accelerated at the FS. The HE band includes a pronounced cut-off region in the spectrum. In all models under consideration the effect of the particle acceleration at the RS vanishes above $1 \mathrm{TeV}$, thus this value is naturally selected as dividing point. Accordingly, the LE band is defined as encompassing $E_{\min }-1 \mathrm{TeV}$ and likewise for the $\mathrm{HE}$ band $1 \mathrm{TeV}-E_{\max }$.

The observed energy spectra in the LE band are fitted with a simple power-law

$$
\frac{d N_{\mathrm{LE}}}{d E}=A_{\mathrm{LE}} \cdot E^{-\alpha_{\mathrm{LE}}}
$$

where $A_{\mathrm{LE}}$ is a normalization constant and $\alpha_{\mathrm{LE}}$ is the power-law index. In the HE band the spectrum is parametrized with a power-law with exponential cutoff:

$$
\frac{d N_{\mathrm{HE}}}{d E}=A_{\mathrm{HE}} \cdot E^{-\alpha_{\mathrm{HE}}} \cdot \mathrm{e}^{-E / E_{\mathrm{c}}},
$$

where $A_{\mathrm{HE}}$ is the normalization constant, $\alpha_{\mathrm{HE}}$ is the power-law index and $E_{\mathrm{c}}$ is the cut-off energy.

In addition, we study the possibility to identify the source type by examining the overall spectral shape. We extract the observed spectral residuals with respect 

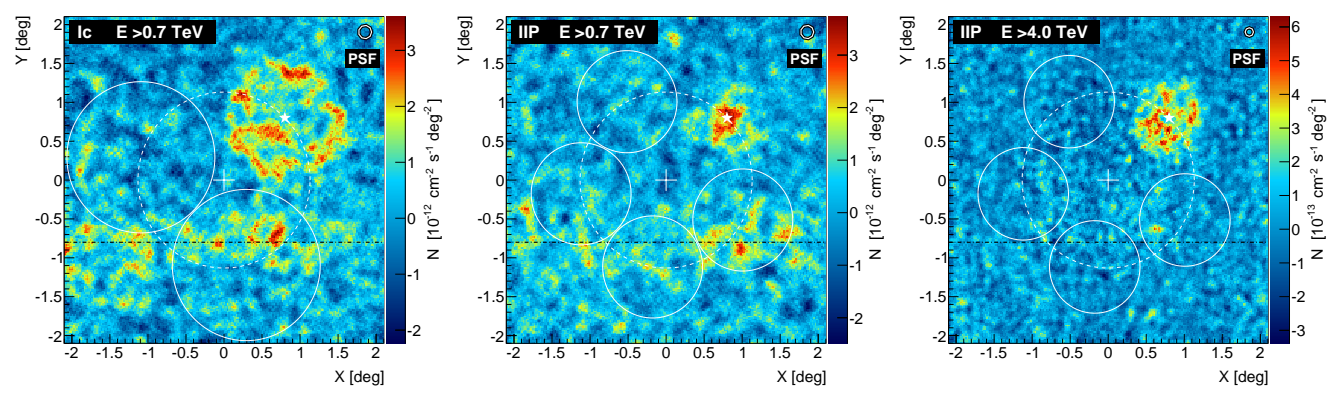

Figure 4: Long exposure (200 hours) $\gamma$-ray images. The meaning of white circles is described in the caption of the Fig. 3 , the white star denote the position of the SNR center and the black dot-dashed line represents the Galactic plane. Left: Gamma-ray image of a 2000 year-old type-Ic SNR at a distance of $1 \mathrm{kpc}$ for energies above $0.7 \mathrm{TeV}$. The contribution of diffuse $\gamma$-ray background concentrated around the Galactic plane is visible. Center: Gamma-ray image of a 2000 yearold type-IIP SNR at $1 \mathrm{kpc}$ distance for energies above $0.7 \mathrm{TeV}$. Right: Gamma-ray image of a 2000 year-old type-IIP SNR at a distance of $1 \mathrm{kpc}$ for energies above $4 \mathrm{TeV}$.

to all theoretical models and calculate the corresponding $\chi^{2}$ values. In this way we obtain the probability that the model in question describes the spectral data points.

\subsection{Morphology studies}

In addition to the resolvability analysis described above we performed more detailed studies on the source morphology. Since the number of $\gamma$-ray events is rather limited we tried to keep the number of parameters for the profile fit as small as possible. This procedure is justified by the fact that the instrument point spread function usually does not allow to distinguish the very fine morphological structure of the source, and complex profile models with a large number of parameters can be verified only for substantially extended and bright sources.

Most of the theoretical profiles of shell-type SNRs have a prominent peak at a certain radius (see the right panel of Fig 3, blue curve). We denote this characteristic radius as $R_{\mathrm{th}}$. In general the position of the peak with respect to the forward shock and the relative brightness of the peak depends on the SNR type and the selected $\gamma$-ray energy band. In some cases the emission of the shell is not powerful enough to develop a peak. In these cases the characteristic radius, $R_{\mathrm{th}}$, is 
defined as the position where the profile brightness reaches $50 \%$ of the brightness at center of the source.

Since all theoretical profiles are the results of numerical simulations there are no analytical expressions for profile shapes. To provide such analytical expression we approximate the complex theoretical profile as the sum of two components: a uniform disk of radius $R_{\text {eff }}$ and a Gaussian that represents a thin shell placed at the same radius $R_{\text {eff }}$. The disk edge is smeared with the error function with sigma parameter $\sigma_{\mathrm{d}}$ and the Gaussian shell has the sigma $\sigma_{\mathrm{s}}$. Considering that, the following expression for the theoretical profile shape is obtained:

$$
I(r)=I_{0} \cdot\left[\operatorname{erf}\left(\frac{R_{\mathrm{eff}}-r}{\sqrt{2 \cdot \sigma_{\mathrm{d}}^{2}}}\right)+\operatorname{erf}\left(\frac{R_{\mathrm{eff}}+r}{\sqrt{2 \cdot \sigma_{\mathrm{d}}^{2}}}\right)+\phi\left(r, \sigma_{\mathrm{s}}\right)\right],
$$

where $I_{0}$ is the normalization factor and the shell term $\phi\left(r, \sigma_{\mathrm{s}}\right)$ is:

$$
\phi\left(r, \sigma_{\mathrm{s}}\right)=\phi_{0} \cdot\left[\exp \left(\frac{-\left(R_{\mathrm{eff}}-r\right)^{2}}{2 \cdot \sigma_{\mathrm{s}}^{2}}\right)+\exp \left(\frac{-\left(R_{\mathrm{eff}}+r\right)^{2}}{2 \cdot \sigma_{\mathrm{s}}^{2}}\right)\right],
$$

where $\phi_{0}$ is the factor describing the relative brightness of the shell.

To obtain the fitting fuction for measured profiles the Eq. 3 is convolved with the known Gaussian instrument PSF with sigma $\sigma_{\text {PSF. This convolution does not }}$ change the form of the Eq. 3 and the final profile fitting function is obtained if the following substitutions are done:

$$
\sigma_{\mathrm{d}} \rightarrow \sqrt{\sigma_{\mathrm{d}}^{2}+\sigma_{\mathrm{PSF}}^{2}}, \quad \sigma_{\mathrm{s}} \rightarrow \sqrt{\sigma_{\mathrm{s}}^{2}+\sigma_{\mathrm{PSF}}^{2}} .
$$

Parameters of the profile fit are $R_{\mathrm{eff}}, \sigma_{\mathrm{d}}, \sigma_{\mathrm{s}}$ and the normalization factors $I_{0}$ and $\phi_{0}$. Here we note that the contribution of the instrument PSF to the profile shape determination can be dominant: $\sigma_{\mathrm{d}, \mathrm{s}}<0.1 \sigma_{\mathrm{PSF}}$, especially for large distances. In such cases we set quantities $\sigma_{\mathrm{d}}$ and $\sigma_{\mathrm{s}}$ to zero to further reduce the number of fitting parameters. Moreover, if the $\gamma$-ray emission from the shell is not significant, the shell component can be neglected, setting $\phi\left(r, \sigma_{\mathrm{s}}\right)=0$. An example of a profile fit is shown on the right panel of Fig 3.

The fitting procedure with Eq. 3 and substitutions defined by Eq. 5 is performed for three energy ranges: $E_{\min }-3 \mathrm{TeV}, 3 \mathrm{TeV}-E_{\max }$, and $E_{\min }-E_{\max }$, where $E_{\min }, E_{\max }$ are the same as in the spectral analysis. The parameter $R_{\text {eff }}$ for the fit with smallest reduced $\chi^{2}$ is then compared with the theoretical radius $R_{\mathrm{th}}$, obtained for the same energy range (see Fig. 6). The delimiting value of $3 \mathrm{TeV}$ 


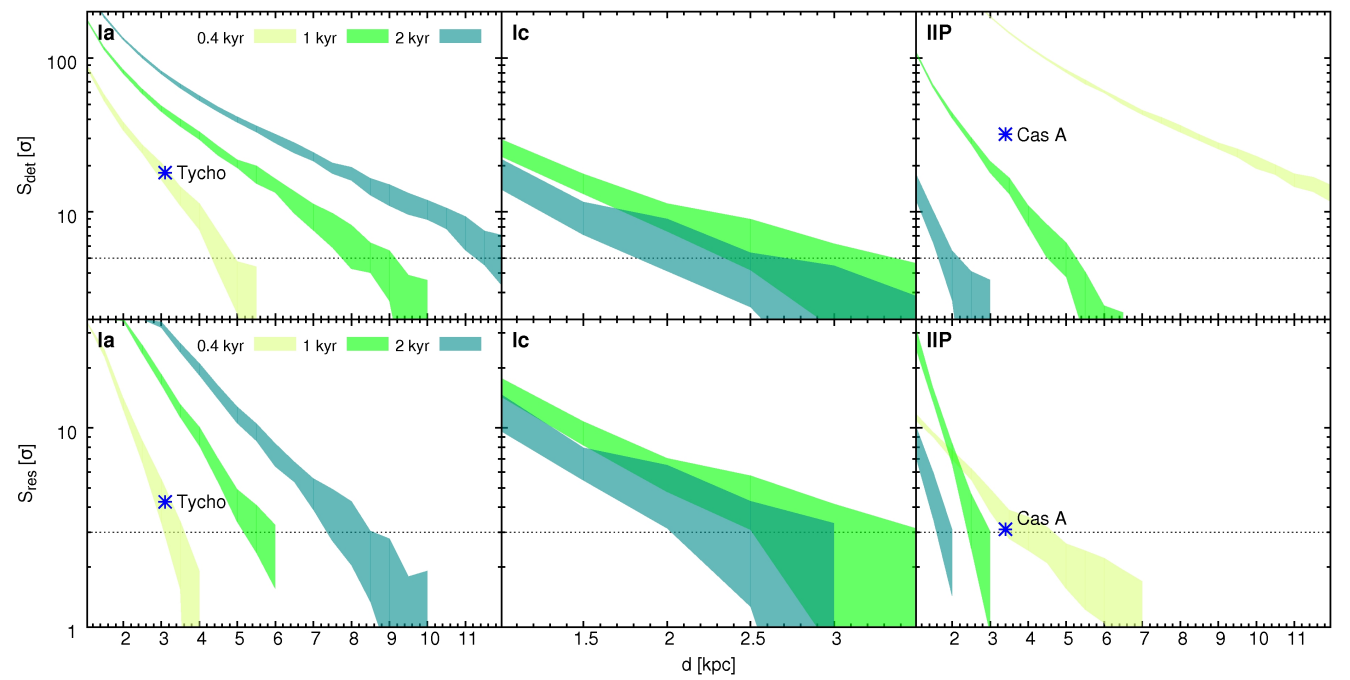

Figure 5: Detection and resolvability significances for 50 hours exposure time. Labels "Tycho" and "Cas A" denote Tycho-like and Cas A-like sources correspondingly. Top: Post-trial detection significance for type-Ia (left), type-Ic (center) and type-IIP (right) SNRs at different ages as a function of distance. Bottom: Resolvability significance for the same types of SNRs. The thresholds for detection $(5 \sigma)$ and resolvability $(3 \sigma)$ of the sources are shown with dotted lines.

was chosen according to the CTA angular resolution, $\Phi_{\text {AngRes }}(\theta, E)$, which significantly improves above $3 \mathrm{TeV}$ for any off-axis angle, $\theta$, as depicted on Fig 2 .

The influence of astrophysical background on morphological studies is demonstrated in Fig 4. For this purpose, we shifted the CTA center of FoV by $0.8 \mathrm{deg}$ from the Galactic plane and simulated an exposure time of 200 hours. If the hadron/electron residual background is estimated only from the upper half of the FoV then the $\gamma$-ray excess pattern of astrophysical background is visible along with the source of comparable intensity. Thus the diffuse emission becomes an issue for faint sources and a careful selection of off-source regions is required.

The increased exposure, as seen from the maps presented at Fig 4 (central and right panels), allows for energy-dependent morphological studies of certain types of SNRs as for example, type-IIP SNRs. In this particular case, the soft emission of pion-decay origin comes from the dense central region of the remnant, whereas the extended high-energy emission is due to IC scatterings of electrons on the cosmic microwave background. 


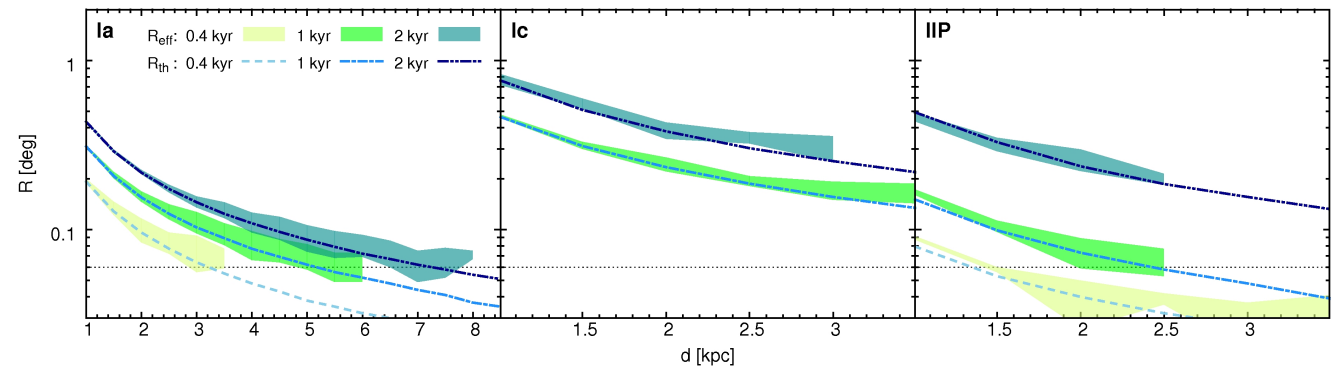

Figure 6: The reconstructed effective radii $R_{\text {eff }}$ of type-Ia (left), type-Ic (center) and type-IIP (right) SNRs at different ages as a function of distance plotted along with theoretical radii $R_{\mathrm{th}}$ (blueish dashed lines) determined from the theoretical profiles. The dotted lines represent the CTA angular resolution at $3 \mathrm{TeV}$ for $1^{\circ}$ offset angle. The exposure time is 50 hours.

\section{Results and discussion}

We simulated CTA data sets for various SNR models, characterized by SNR type and age, considered distances in the range from 1.0 to $12.0 \mathrm{kpc}$ with steps of $0.5 \mathrm{kpc}$. The observation time was set to 50 hours, and the zenith angle was assumed to be $20^{\circ}$. Data analysis was performed as described in Section 4 . We repeated all simulations with subsequent data analysis 20 times to reduce the weight of a specific random realization. All results presented are the mean values over these realizations, the error bands show the corresponding one sigma standard deviation. Reconstructed parameters (i.e., radii, spectral indices and cut-off energies curves) are removed from the analysis if less than $30 \%$ of the random realizations return an estimated value with a relative error less than $50 \%$. Since the parameters chosen for the SNR models are generic, our results demonstrate a general assessment of the parameter space for a given SNR type to be probed with CTA.

We note that the analysis in the LE band is strongly constrained by the CTA sensitivity at low energies. This leads to a fast shrinking of the measurable energy range with source distance (brightness). Thus, the LE band can be covered by CTA for nearby sources only. However, the sensitivity at low energies is being continuously revised and will be improved by means of advanced analysis methods (Becherini et al., 2012; Shayduk \& CTA Consortium, 2013). One should keep in mind that the CTA layout optimization for high energies, as well as the observations with large zenith angles (Konopelko et al., 1999) may slightly improve the cut-off energies determination, especially for SNRs with 


\section{hard spectra, but the LE band results would be further deteriorated.}

\subsection{Detectability and resolvability of the SNRs}

The detectability of a SNR model is defined by a $5 \sigma$ detection threshold. We define the resolvability of a SNR model with a $3 \sigma$ confidence threshold for pointsource hypothesis rejection. The detectability and resolvability plots for various SNR types at different ages are presented in Fig. 5.

The type Ia SNR models considered here are sources dominated by pion-decay emission. This is a result of the assumed ISM number density of $\simeq 0.4 \mathrm{~cm}^{-3}$. As mentioned above, the RS contribution to the accelerated particles becomes unimportant very quickly as type-Ia SNRs evolve (Telezhinsky et al., 2012a), so the emission spectra are power-laws with indices $\alpha \simeq 2$ and exponential cutoffs (see Fig 1, left panel). As demonstrated in Fig. 5, type-Ia SNRs are bright sources for CTA. Even at the very young age of 400 years (close to the age of Tycho SNR) we would be able to detect it up to $\simeq 5 \mathrm{kpc}$. As a type Ia SNR becomes older, it sweeps more ISM material while the density of CRs inside the FS stays nearly constant. This results in a higher luminosity extending the detectability horizon up to $\simeq 10 \mathrm{kpc}$ for a 1000 -year-old SNR and $\geq 12 \mathrm{kpc}$ for the 2000 -year-old remnant. As expected, the resolvability horizons are much closer than those of detectability. A Tycho-like type-Ia SNR would be resolved out to distance of $\simeq 3.5 \mathrm{kpc}$. Older type-Ia SNRs will be resolved out to $\simeq 6$ and $\simeq 8.5 \mathrm{kpc}$ at the age of 1000 and 2000 years, respectively.

Type-Ic SNRs should generally be rather weak sources of pion-decay emission (Telezhinsky et al., 2012b, 2013), if there is no significant emission from the windblown shells of CSM material. The reason is that type-Ic SNRs evolve in the wind-blown cavities of progenitor stars. The density in the cavity follows a powerlaw profile out to the termination shock, which can be located 5-10 pc from the explosion center. The density profile beyond this is nearly flat and very low in value, $n \sim 0.001 \mathrm{~cm}^{-3}$. The number density of CRs, which is proportional to the amount of injected particles from the shock-heated CSM, is therefore very low. Under these conditions, the IC emission dominates over pion-decay, and the expected spectra are hard. In our model of type-Ic SNR, the complex plasma profiles result in enhanced acceleration of particles at the RS around an age of 1000 years (details can be found in Telezhinsky et al., 2013). After that point in time an additional significant contribution of IC emission in the LE band is visible in the middle panel of Fig 1, which spreads to higher energies as the SNR ages. The total radiation flux of type-Ic SNR grows with time as the size of the ICemission zone increases. It is detected only at late ages of 1000 and 2000 years as 
shown in Fig 5. The detection significance, though, is not strikingly high, and the sources fade out if they are located further than $4 \mathrm{kpc}$. The expansion in a rarefied medium makes type-Ic SNRs very large in radius, and therefore one expects their resolvability and detectability horizons to be similar.

Type-IIP SNRs are very different from type-Ia and type-Ic remnants. Early on, they expand in a very dense RSG wind that terminates a few parsec away from the SNR center. After leaving the dense RSG wind zone, the FS propagates through the dilute main-sequence wind of the progenitor star and accelerates CRs to higher energies than before because its velocity increases while the number of injected particles sharply drops. Meanwhile, the RS travels inwards through still dense ejecta. This produces an additional population of low-energy CRs accelerated at the RS (see Fig. 1, right panel). Since the ejecta is very dense, the dominant emission process is pion-decay and the resulting spectra are soft. When the FS is sufficiently far from the RS and has encompassed a large volume of dilute medium, the IC contribution in the HE band becomes apparent (see Fig 1, right panel). The number density of CRs decreases as the SNR expands, and so the radiation flux decreases as well. Since the source spectrum is soft and the SNR is very bright during the first thousand years, it can be well detected not only in the HE band, but also in the LE band. At the age of 2000 years it fades away. The emission region in type-IIP SNRs is then compact with a radius of a few parsec, so that it becomes minuscule in angular size at large distances. Nonetheless, young type-IIP SNRs are resolved up to distance of $\simeq 4 \mathrm{kpc}$, provided that systematic uncertainties of PSF determination remain below the level of $5 \%$.

\subsection{Reconstructing the radius of SNRS}

Among the parameters that could be used to constrain theoretical models are the source radii at various wavelengths. Radio, optical, and X-ray instruments can measure the leptonic population of cosmic rays much more precisely than the current Cherenkov telescopes, which can resolve only a handful of objects. Finding correlations or discrepancies in source radii between $\gamma$-rays and other wavebands would be extremely useful to identify the type of radiating particles.

Here we attempt to reconstruct the radii of various SNR models in the $\gamma$ ray band with CTA instrument. The data analysis including the various fitting procedures for the source radius is described in Sec. 4.2.

The effective SNR radii were reconstructed as follows. We selected cases for which the point-source hypothesis was rejected at the $3 \sigma$ level. Then we fit intensity profiles with Eq. 3 as described in Sec. 4.4 for all energy bands and choose the best fit by comparing $\chi^{2}$ values. The fit parameter, $R_{\mathrm{eff}}$, represents the 


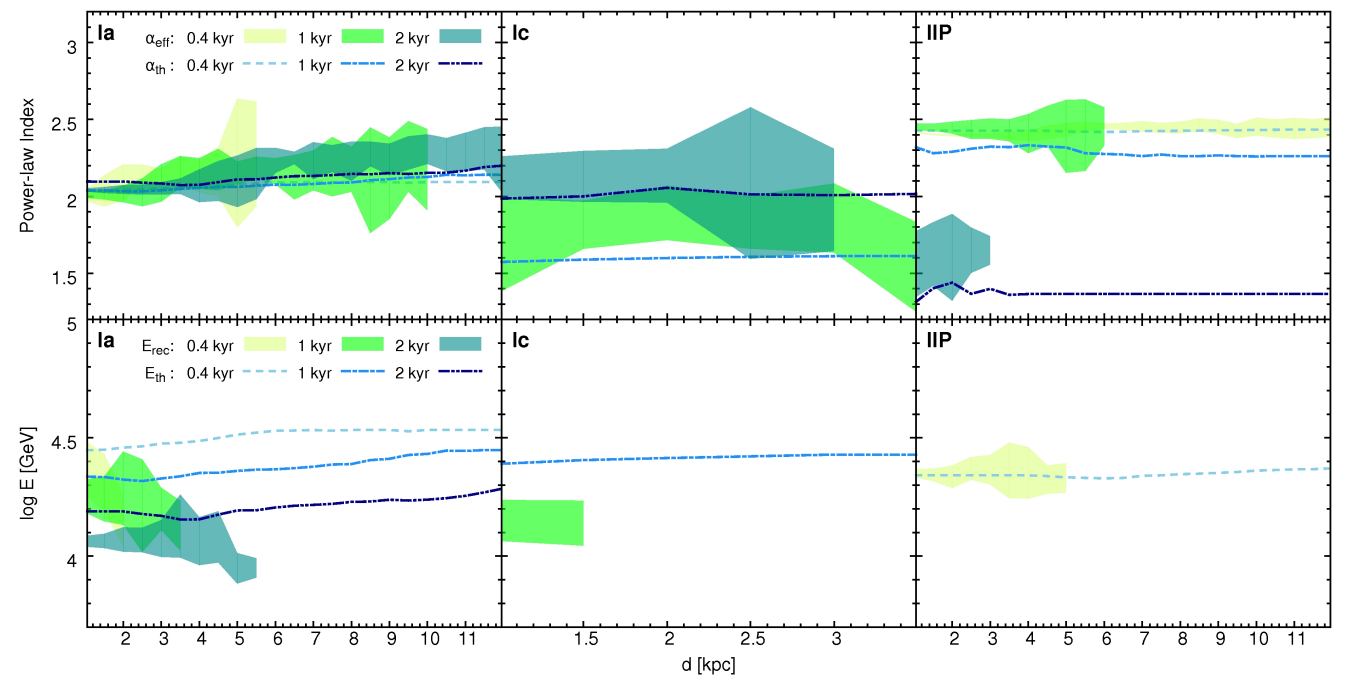

Figure 7: Top: The reconstructed power-law indices, $\alpha_{\text {eff }}$, of type-Ia (left), typeIc (center) and type-IIP (right) SNRs at different ages as a function of distance plotted along with theoretical values, $\alpha_{t h}$, (Dashed lines). The theoretical value is determined by fitting theoretical spectra in the same energy band as the simulated data. Bottom: The reconstructed cut-off energies of type-Ia (left), type-Ic (center) and type-IIP (right) SNRs at different ages.

reconstructed effective SNR radius. The results for $R_{\text {eff }}$ compared to theoretical values $R_{\text {th }}$ are presented at Fig 6 .

We find that out to the resolvability horizons the radii are well reconstructed for all SNR models. One exception are 400-year-old type-IIP SNRs. Despite the very small angular size, their good resolvability was provided by their substantial brightness. It allowed to measure small deviations from the intensity profile a point source, even if the radius fell below the PSF size.

It is worth noting that the radius of maximum cumulative significance, $R_{\mathrm{MS}}$, roughly corresponds to the intrinsic source radius, $R_{\text {th }}$ (see Fig 3 ). It can therefore be used as a robust estimate for the radius even if the source is poorly resolved, provided the center position is known from information at other wavelengths.

\subsection{Spectral indices and cut-off energies}

The primary aim of the majority of observations with CTA will be to obtain precise spectral measurements as opposed to the mainly discovery-motivated observations of current instruments. Detailed high-energy $\gamma$-ray spectra are impor- 
tant to constrain theoretical models. In this section we examine the ability of CTA to measure spectral parameters of SNRs located at different distances.

The procedure to reconstruct spectral parameters is given in Sec. 4.3. We selected only models detected above the $5 \sigma$ threshold. For a fit to the simulated data with a power-law plus exponential cutoff to be favored over a simple powerlaw fit, not only must the reduced $\chi^{2}$ be smaller, but also the relative uncertainty of the cutoff energy should be less than $30 \%$ to make it suitable for constraining models. The theoretical spectra were fitted with the same procedures in the same energy bands and the same binning as the simulated experimental data to derive the corresponding theoretical indices and cut-off energies.

The indices found for the LE band do not satisfactorily reproduce the theoretical values. They have a large scatter which significantly increases with distance. This means that most of the intriguing spectral features of SNRs, such as the emission from the RS region which shows up in the LE band, may not be detectable with CTA.

Identifying cut-off energies in $\gamma$-ray radiation of young SNRs is extremely important for finding Galactic PeVatrons. If some of the SNRs are indeed PeVatrons, one should be able to measure cut-off energies of at least $100 \mathrm{TeV}$, which seems rather difficult with the current CTA sensitivity and 50 hours of exposure. This is due to low event statistics at high energies that could be improved with significantly longer exposures. Our results for indices and cut-off energies are shown at Fig 7.

Type-Ia SNRs prove to be the best-observable sources. The spectral index is nearly constant ( $\alpha \simeq 2$ ) with time, it is well reconstructed up to 5, 10, and $12 \mathrm{kpc}$ for SNRs with ages of 400, 1000, and 2000 years, respectively. Their cut-off energy decreases with SNR age. Due to low statistics at the highest energies, the horizons for measuring cut-off energies are much closer. The spectral indices and cut-off energies are rather well reproduced. We will likely be able to determine the spectral index of Tycho's SNR, but would probably be unable to find the cut-off energy (at least with 50 hours exposure time).

Type-Ic SNRs are dim, which limits our ability to reconstruct their spectral parameters. It will be challenging for CTA to measure the spectral index of very young type-Ic SNR. Taking into account that the sources are faint means we probe the spectra in the region of maximum sensitivity around $1 \mathrm{TeV}$, thus the contribution of the RS-accelerated particles to the emission is not possible to establish. However, the intrinsic theoretical spectral indices of 1000- and 2000-years-old SNRs are different also above $1 \mathrm{TeV}$. We can distinguish the two indices up to the distance of $\simeq 2 \mathrm{kpc}$. Further away, the obtained values are ambiguous. The cut-off 
energy can be reconstructed only for a 1000-year-old and very nearby SNR. The measured value, however, is strongly underestimated.

A prominent young type IIP SNR would be visible with high significance at very large distances, which makes measurements of its spectral characteristics with CTA an easy task. It is a very soft and bright source, so even the LE-band component is measurable at all distances. The spectral index is well measured, and so is the cut-off energy. At the age of 1000 years, type-IIP SNRs are no longer bright and the spectra slightly harden at high energy due to the IC contribution. Finally, at the age of 2000 years, type-IIP SNRs fade away. Very-high-energy radiation, which CTA probes, is completely dominated by IC emission, which means that the spectral index is hard. Unfortunately, we can measure it only for very nearby SNR (see Fig. 7). The determination of the cut-off energies is not possible for type-IIP SNRs at the age of 1000 and 2000 years for an exposure of 50 hours.

\subsection{Unveiling the underlying SNR model by measuring energy spectra}

Measurements by CTA will allow to constrain theories of $\gamma$-ray emission from SNRs. In this section we derive the horizons for spectral measurements that permit to distinguish theoretical models of SNRs. For this we fit the reconstructed spectral shape of a model, which we call the reference model, with the theoretical shapes of all models considered in this paper. We also compare to variations of models in which CR acceleration at the RS is not considered. We repeat this procedure for all distances. The fit probability should be highest for the spectrum of the reference model. The probability value serves as a measure of how well the data constrain or reject all other theoretical spectra. The discrimination power deteriorates with distance. The distance where multiple models fit the data can be considered as the horizon of acceptable model discrimination for the given SNR type and age. Beyond this horizon the data are not constraining. We show and discuss the most interesting cases shown in Fig 8, where each panel corresponds to a reference model. The Y-axis lists SNR models characterized by type and age used to fit the reconstructed spectrum of the reference model. A prime (') in the model name marks the neglect of CR acceleration at the RS. The X-axis is the distance to the source. The shaded region indicates that the reference source is not detected.

Tycho-like SNRs (Young type-Ia remnants, model "Ia 0400", the top-left panel of Fig. 8) at $1 \mathrm{kpc}$ can be clearly identified, as shown by the high probability for fits with their own theoretical spectrum. However, it appears impossible to distinguish type-1a models with and without CR acceleration at the reverse shock. Beyond a 
distance of $3 \mathrm{kpc}$ (approx. the distance of Tycho), $\gamma$-ray data no longer permit the identification of the type and age of the remnant. In contrast to very young type-Ia SNRs, the much brighter 2000 year-old remnants are clearly distinguished from other models up to $\simeq 5 \mathrm{kpc}$. It appears that especially the type-Ic models, which are IC dominated, are very poor fits.

The faint type-Ic SNRs are the most challenging objects. It will not be possible to measure the contribution of the RS-accelerated particles to the emission of a 1000-year-old SNR, even if it is only $1 \mathrm{kpc}$ away. At a distance of $2 \mathrm{kpc}$ one can fit the measured spectrum of this SNR type with model spectra of type-Ia and middle-age type IIP SNRs. A 2000-year-old type Ic remnant is also likely misidentified as young type-Ia object.

The best discrimination power is found for very bright 400-year-old type-IIP SNRs, for which the contribution of RS-accelerated particles can be firmly established. Up to a distance of $6 \mathrm{kpc}$ all other theoretical spectra are ruled out. Further away they can also be fitted with models of the older remnants of the same type, indicating that the spectra in the energy range probed by CTA simply do not change enough with age to permit discrimination. A similar result is observed with 1000 year-old remnants, but since they are much dimmer, confusion sets in much earlier at a distance of about $3.5 \mathrm{kpc}$.

\section{Conclusions}

We addressed the potential of the Cherenkov Telescope Array to study young SNRs. In this paper we covered a number of questions ranging from simple detection to constraints on theoretical models. We provide the scientific community with an understanding to what degree the next generation $\gamma$-ray observatory CTA can detect spectral and morphological features of SNRs. Our work also provides guidance in which way the instrument should be improved to optimize the scientific return.

We note that there is no standard young SNR. There are large variations in spectra and morphology between different remnants. The best observable SNRs for CTA are of type-Ia. Older remnants are more clearly detected and resolved better. The spectral and morphology measurements will be of very high quality. Type-Ia remnants that are a few thousand years old can be seen anywhere in the Galaxy provided they reside in ISM of at least average density. Younger SNRs are weaker emitters and not as easily detected. Of course, the parameter range for type-Ia SNR may vary. If it exploded in a denser medium it would be brighter, 
more compact and better detectable. However, the parameter range for density variation is not large, so the trend we find here is realistic.

The next best class of SNRs for observations with CTA are remnants of corecollapse type-IIP explosions, but only if they are very young. They are extremely luminous and will be detectable throughout the Galaxy. The small size of the emitting region makes them hard to resolve, requiring a low systematic uncertainty of the instrument. The spectral parameters of young type IIP SNRs will be measured extremely well. Cas A resembles this class of objects very well, as it is also very bright and compact with indications of CR acceleration at the RS (Gotthelf et al., 2001; Helder \& Vink, 2008). It will become a good theory testbed with CTA. As type-IIP SNRs age, their luminosity drops sharply and all detection horizons shrink quickly, so CTA will very likely not detect any type-IIP SNRs older than several thousand years.

The most challenging objects are the dim type-Ic SNRs. The horizons for their detectability, resolvability, spectral and morphological reconstruction are nearby, within a few kpc. We suspect that the astrophysical background and confusion play an important role for the analysis of these faint sources for CTA.

We note that we used generic parameters for core-collapse SNRs, and there might be some variation in the gas density in the progenitor wind zones. To zeroth order that should only scale in time the spectral and morphological evolution of the remnants, and therefore we estimate as not very strong its effect on the reconstruction of the physical characteristics of SNR with CTA data. The general trend seen in all core-collapse SNR models makes us conclude that they will not be seen by CTA after several thousand years of evolution at distances further than a few kpc. This places a strong limit on the number of core-collapse SNRs detectable by CTA. Those that are seen would provide invaluable information, though. In contrast, with present instrumentation it is very difficult, if not impossible, to determine whether or not Cas A is an extended emitter of $\gamma$-rays and how efficient $\mathrm{CR}$ acceleration at the $\mathrm{RS}$ is.

An interesting aspect of core-collapse supernovae that would require a dedicated study is emission from the shell of CSM material swept-up by the wind of the progenitor star (Telezhinsky et al., 2012b). This may be the best way to detect older core-collapse SNRs in very high-energy $\gamma$-rays. The shell may be located far from the explosion center and the diffusion properties of the medium will play a crucial role in spectral evolution of the emission. When the core-collapse SNR is young, the CSM shell is not bright. The FS is far from it and CRs have to propagate there to illuminate it. However, if the SNR is old enough, the emission comes predominantly from the CSM shell and not from the remnant, and so spectral and 
morphological properties of the source should be completely different. Particularly intriguing would the possibility to infer the efficacy of turbulence generation by CRs between the FS and the shell, which is a critical but poorly known input parameter in models of CR acceleration at SNR shocks.

\section{Acknowledgements}

We gratefully acknowledge financial support from the following agencies and organizations:

Ministerio de Ciencia, Tecnología e Innovación Productiva (MinCyT), Comisión Nacional de Energía Atómica (CNEA), Consejo Nacional de Investigaciones Científicas y Técnicas (CONICET), Argentina; State Committee of Science of Armenia, Armenia; Conselho Nacional de Desenvolvimento Científico e Tecnológico (CNPq), Fundação de Amparo à Pesquisa do Estado do Rio de Janeiro (FAPERJ), Fundação de Amparo à Pesquisa do Estado de São Paulo (FAPESP), Brasil; Croatian Science Foundation, Croatia; Ministry of Education, Youth and Sports, MEYS LE13012, 7AMB12AR013, Czech Republic; Ministry of Higher Education and Research, CNRS-INSU and CNRS-IN2P3, CEA-Irfu, ANR, Regional Council Ile de France, Labex ENIGMASS, OSUG2020 and OCEVU, France; Max Planck Society, BMBF, DESY, Helmholtz Association, Germany; Department of Atomic Energy, Department of Science and Technology, India; Istituto Nazionale di Astrofisica (INAF), MIUR, Italy; ICRR, University of Tokyo, JSPS, Japan; Netherlands Research School for Astronomy (NOVA), Netherlands Organization for Scientific Research (NWO), Netherlands; The Bergen Research Foundation, Norway; Ministry of Science and Higher Education, the National Centre for Research and Development and the National Science Centre, Poland; MINECO support through the National $\mathrm{R}+\mathrm{D}+\mathrm{I}$, CDTI funding plans and the CPAN and MultiDark Consolider-Ingenio 2010 programme, Spain; Swedish Research Council, Royal Swedish Academy of Sciences, Sweden; Swiss National Science Foundation (SNSF), Ernest Boninchi Foundation, Switzerland; Durham University, Leverhulme Trust, Liverpool University, University of Leicester, University of Oxford, Royal Society, Science and Technologies Facilities Council, UK; U.S. National Science Foundation, U.S. Department of Energy, Argonne National Laboratory, Barnard College, University of California, University of Chicago, Columbia University, Georgia Institute of Technology, Institute for Nuclear and Particle Astrophysics (INPAC-MRPI program), Iowa State University, Washington University McDonnell Center for the Space Sciences, USA. 
The research leading to these results has received funding from the European Union's Seventh Framework Programme (FP7/2007-2013) under grant agreement No. 262053.

Acero, F., Bamba, A., Casanova, S., et al. 2013, Astroparticle Physics, 43, 276

Acharya, B. S., Actis, M., Aghajani, T., et al. 2013, Astroparticle Physics, 43, 3

Ackermann, M., Ajello, M., Allafort, A., et al. 2013, Science, 339, 807

Actis, M., Agnetta, G., Aharonian, F., et al. 2011, Experimental Astronomy, 32, 193

Adriani, O., Barbarino, G. C., Bazilevskaya, G. A., et al. 2011, Science, 332, 69

Apel, W. D., Arteaga-Velázquez, J. C., Bekk, K., et al. 2012, Astroparticle Physics, 36, 183

Becherini, Y., Khélifi, B., Pita, S., Punch, M., \& CTA Consortium. 2012, in American Institute of Physics Conference Series, Vol. 1505, American Institute of Physics Conference Series, ed. F. A. Aharonian, W. Hofmann, \& F. M. Rieger, 769-772

Berezhko, E. G. \& Völk, H. J. 1997, Astroparticle Physics, 7, 183

Berge, D., Funk, S., \& Hinton, J. 2007, A\&A, 466, 1219

Bernlöhr, K., Barnacka, A., Becherini, Y., et al. 2013, Astroparticle Physics, 43, 171

Blasi, P., Gabici, S., \& Vannoni, G. 2005, MNRAS, 361, 907

Bretz, T., Dorner, D., Riegel, B., Höhne, D., \& Berger, K. 2005, in International Cosmic Ray Conference, Vol. 4, International Cosmic Ray Conference, 311

Bykov, A. M., Ellison, D. C., Osipov, S. M., \& Vladimirov, A. E. 2014, ApJ, 789, 137

Caprioli, D., Blasi, P., Amato, E., \& Vietri, M. 2009, MNRAS, 395, 895

Caprioli, D., Kang, H., Vladimirov, A. E., \& Jones, T. W. 2010, MNRAS, 407, 1773 
Chevalier, R. A. \& Fransson, C. 1994, ApJ, 420, 268

Chevalier, R. A. \& Oishi, J. 2003, ApJ, 593, L23

Dame, T. M., Hartmann, D., \& Thaddeus, P. 2001, ApJ, 547, 792

Daum, A., Hermann, G., Hess, M., et al. 1997, Astroparticle Physics, 8, 1

Dwarkadas, V. V. 2005, ApJ, 630, 892

Dwarkadas, V. V. 2007, ApJ, 667, 226

Dwarkadas, V. V. \& Chevalier, R. A. 1998, ApJ, 497, 807

Ferrand, G., Decourchelle, A., \& Safi-Harb, S. 2014, ApJ, 789, 49

Funk, S., Hinton, J. A., \& CTA Consortium. 2013, Astroparticle Physics, 43, 348

Gibson, S. J., Taylor, A. R., Higgs, L. A., Brunt, C. M., \& Dewdney, P. E. 2005, ApJ, 626, 214

Ginzburg, V. L. \& Syrovatsky, S. I. 1961, Progress of Theoretical Physics Supplement, 20, 1

Gotthelf, E. V., Koralesky, B., Rudnick, L., et al. 2001, ApJ, 552, L39

Helder, E. A. \& Vink, J. 2008, ApJ, 686, 1094

Huang, C., Park, S., Pohl, M., \& Daniels, C. D. 2007, Astroparticle Physics, 27, 429

Kalberla, P. M. W., Burton, W. B., Hartmann, D., et al. 2005, A\&A, 440, 775

Kang, H., Jones, T. W., \& Edmon, P. P. 2013, ApJ, 777, 25

Konopelko, A., Aharonian, F., Hemberger, M., et al. 1999, Journal of Physics G Nuclear Physics, 25, 1989

Lee, S.-H., Ellison, D. C., \& Nagataki, S. 2012, ApJ, 750, 156

Li, T.-P. \& Ma, Y.-Q. 1983, apj, 272, 317

McClure-Griffiths, N. M., Dickey, J. M., Gaensler, B. M., et al. 2005, ApJS, 158, 178 
Menn, W., Hof, M., Reimer, O., et al. 2000, ApJ, 533, 281

Pohl, M., Englmaier, P., \& Bissantz, N. 2008, ApJ, 677, 283

Ptuskin, V., Zirakashvili, V., \& Seo, E. 2010, ApJ, 718, 31

Ptuskin, V., Zirakashvili, V., \& Seo, E.-S. 2013, ApJ, 763, 47

Renaud, M. \& CTA Consortium. 2011, Mem. Soc. Astron. Italiana, 82, 726

Schure, K. M., Achterberg, A., Keppens, R., \& Vink, J. 2010, MNRAS, 406, 2633

Shayduk, M. \& CTA Consortium. 2013, ArXiv e-prints

Smartt, S. J., Eldridge, J. J., Crockett, R. M., \& Maund, J. R. 2009, MNRAS, 395, 1409

Stecker, F. W. 1973, ApJ, 185, 499

Telezhinsky, I., Dwarkadas, V., \& Pohl, M. 2012a, Astroparticle Physics, 35, 300

Telezhinsky, I., Dwarkadas, V. V., \& Pohl, M. 2012b, A\&A, 541, A153

Telezhinsky, I., Dwarkadas, V. V., \& Pohl, M. 2013, A\&A, 552, A102

Zirakashvili, V. N. \& Ptuskin, V. S. 2008, ApJ, 678, 939

Zirakashvili, V. N. \& Ptuskin, V. S. 2011, ArXiv e-prints 1109.4482 

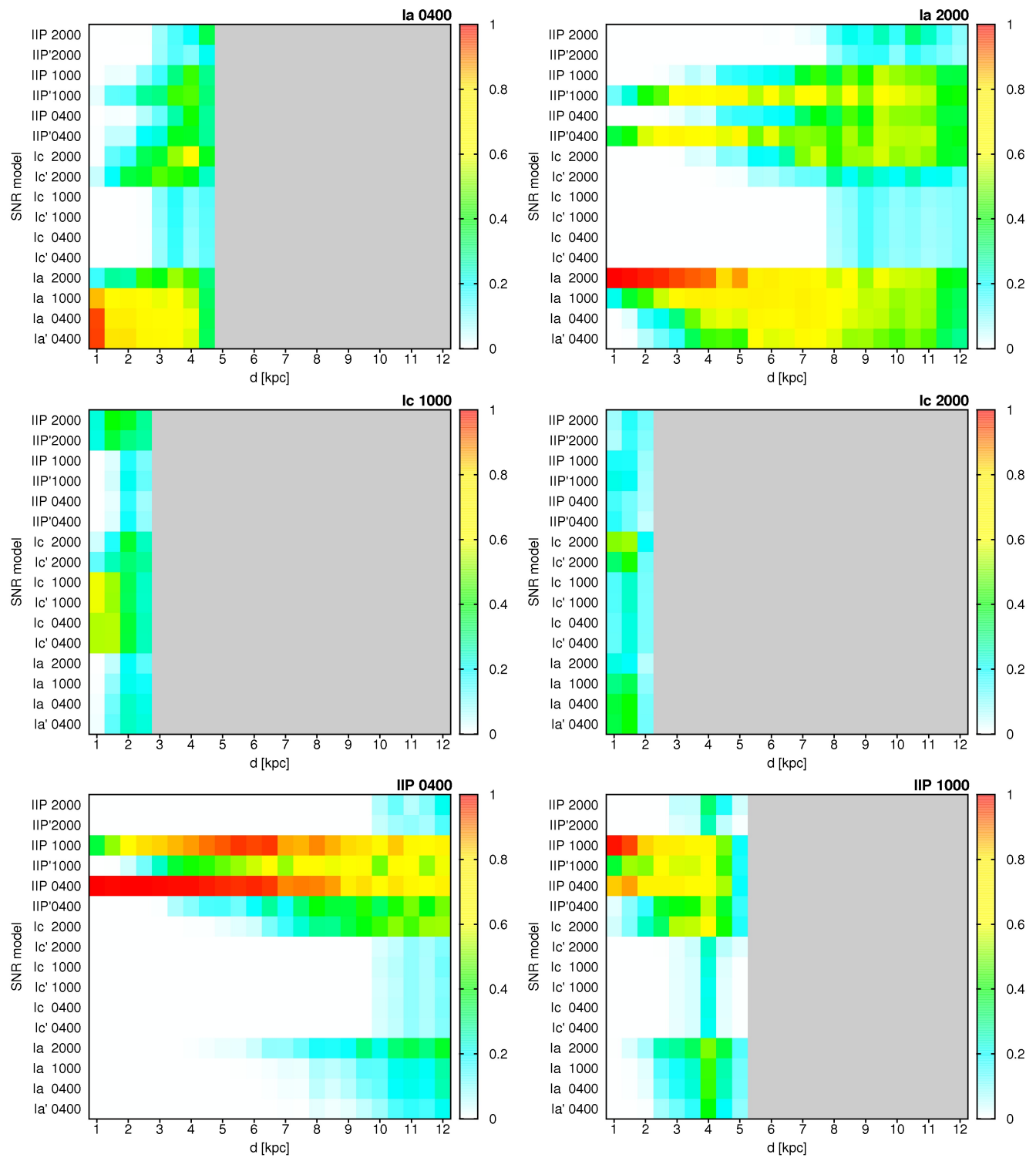

Figure 8: False-color representation of the probability of fitting the experimentally obtained spectrum of a given SNR model with the theoretical spectra of the other SNR models (listed on Y-axis) versus the distance (X-axis). Gray color means there is no data. A prime in the model name indicates that acceleration at the reverse shock is neglected. Top: Type-Ia SNR at the ages of 400 (left) and 2000 (right) years. Middle: Type-Ic SNR at the ages of 1000 (left) and 2000 (right) years. Bottom: Type-IIP SNR at the ages of 400 (left) and 1000 (right) years. 